\title{
Testing the Larson relations in massive clumps
}

\author{
A. Traficante, ${ }^{1 \star}$ A. Duarte-Cabral, ${ }^{2}$ D. Elia, ${ }^{1}$ G. A. Fuller,${ }^{3}$ M. Merello,,${ }^{1}$ S. Molinari,${ }^{1}$ \\ N. Peretto, ${ }^{2}$ E. Schisano ${ }^{1}$ and A. Di Giorgio ${ }^{1}$ \\ ${ }^{1}$ IAPS-INAF, via Fosso del Cavaliere, 100, I-00133 Roma, Italy \\ ${ }^{2}$ School of Physics and Astronomy, Cardiff University, Queens Buildings, The Parade, Cardiff CF24 $3 A A$ \\ ${ }^{3}$ Jodrell Bank Centre for Astrophysics, School of Physics and Astronomy, University of Manchester, Oxford Road, Manchester M13 9PL
}

Accepted 2018 March 22. Received 2018 March 22; in original form 2017 October 13

\begin{abstract}
We tested the validity of the three Larson relations in a sample of 213 massive clumps selected from the Herschel infrared Galactic Plane (Hi-GAL) survey, also using data from the Millimetre Astronomy Legacy Team $90 \mathrm{GHz}$ (MALT90) survey of 3-mm emission lines. The clumps are divided into five evolutionary stages so that we can also discuss the Larson relations as a function of evolution. We show that this ensemble does not follow the three Larson relations, regardless of the clump's evolutionary phase. A consequence of this breakdown is that the dependence of the virial parameter $\alpha_{\text {vir }}$ on mass (and radius) is only a function of the gravitational energy, independent of the kinetic energy of the system; thus, $\alpha_{\text {vir }}$ is not a good descriptor of clump dynamics. Our results suggest that clumps with clear signatures of infall motions are statistically indistinguishable from clumps with no such signatures. The observed non-thermal motions are not necessarily ascribed to turbulence acting to sustain the gravity, but they might be a result of the gravitational collapse at the clump scales. This seems to be particularly true for the most massive $\left(M \geq 1000 \mathrm{M}_{\odot}\right)$ clumps in the sample, where exceptionally high magnetic fields might not be enough to stabilize the collapse.
\end{abstract}

Key words: surveys -stars: formation -stars: kinematics and dynamics-stars: massivestars: statistics - infrared: stars.

\section{INTRODUCTION}

Massive star-forming regions are dominated by highly supersonic non-thermal motions. Velocity dispersions in giant molecular clouds (GMCs; size $\simeq 5-100$ pc; Solomon et al. 1987), massive clumps (regions with size $\simeq 0.5-2$ pc; e.g. Urquhart et al. 2014; Traficante et al. 2015a; Elia et al. 2017) and massive cores (size $\simeq 0.1 \mathrm{pc}$; Zinnecker \& Yorke 2007) are of the order of $1-10 \mathrm{~km} \mathrm{~s}^{-1}$, significantly higher than thermal motions $\left(\simeq 0.25 \mathrm{~km} \mathrm{~s}^{-1}\right.$ for hydrogen molecules at a typical temperature of $T=15 \mathrm{~K})$.

In pioneering work, Larson (1981) investigated these motions in GMCs using the available ${ }^{12} \mathrm{CO}$ data and found that the non-thermal motions can be ascribed to internal turbulence acting to sustain the clouds against gravitational collapse. Larson (1981) showed that molecular clouds follow three fundamental relations.

(i) A size-linewidth power-law relation, which states that in molecular clouds the velocity dispersion $\sigma$ scales proportionally to the radius $R$. The first relation found by Larson was $\sigma \propto R^{0.38}$. Later, the analysis was refined and the relation modified to $\sigma \propto R^{0.5}$ (e.g. Heyer \& Brunt 2004). (ii) Clouds are in approximately virial equilibrium, with a virial parameter $\alpha_{\mathrm{vir}}=E_{k} / E_{G}=5 \sigma^{2} R / G M \simeq 1$, where $M$ is the mass of the region and $G$ is the gravitational constant. This relation implies that the kinetic energy of the system $E_{k} \propto \sigma^{2} M$ is of similar intensity as the gravitational energy of the system, $E_{G} \propto M^{2} / R$.

(iii) A volume density $n$-size relation, $n \propto R^{-1.1}$. This relation implies that GMCs are universal structures, with a mostly uniform column density. From this relation, it follows that the surface density $\Sigma$ is almost constant: $\Sigma \propto R^{-0.1}$.

Early observations of GMCs confirm the validity of the three relations (e.g. Solomon et al. 1987; Heyer \& Brunt 2004), which were also observed in simulations of the turbulent interstellar medium (see Mac Low \& Klessen 2004; McKee \& Ostriker 2007, and references therein).

However, these relations have been questioned over the years. For example, the validity of the third relation was attributed to selection effects (e.g. Kegel 1989). The first and third Larson relations in GMCs were questioned by, for example, Heyer et al. (2009). This work reanalysed the GMCs using ${ }^{13} \mathrm{CO}$ data taken from the Boston University-Five College Radio Astronomy Observatory (FCRAO) Galactic Ring Survey (Jackson et al. 2006). The higher critical density of ${ }^{13} \mathrm{CO}$ compared to ${ }^{12} \mathrm{CO}$ allowed us to trace higher column density regions and these data demonstrate that the 
quantity $\sigma / R^{0.5}$ and the surface density of GMCs are not constant. Nevertheless, the average value of the virial parameter in GMCs, $\alpha_{\text {vir }}=1.9$, is still consistent with virial equilibrium (Heyer et al. 2009).

Challenging one of the Larson relations has direct consequences on the other two relations as well: the three relations are algebraically linked. If two of them are true, then the third is automatically implied (e.g. Kritsuk, Lee \& Norman 2013). At the same time, if one of the three is violated, necessarily (at least) one of the other two relations must not be true, with important implications for different star formation theories. The Larson relations are in fact assumed in models that predict the formation of massive stars through turbulent-regulated collapse (e.g. McKee \& Tan 2003), as opposed to gravity-dominated, almost free-fall, collapse in which the theories predict, for example, values of the virial parameter $\alpha_{\text {vir }}<1$ (e.g. Bonnell, Vine \& Bate 2004).

While the validity of the Larson relations has been widely investigated in GMCs, few and relatively small surveys have been dedicated to the study of non-thermal motions in massive starforming clumps and cores. For example, Ballesteros-Paredes et al. (2011) showed that the first and third Larson relations are violated in GMCs and massive clumps. Simulations of star-forming regions showed that ensembles of clouds, clumps and cores do not follow the three Larson relations, and this is particularly true for the higher-density regions (Camacho et al. 2016). In a recent work, we combined a survey of 16 massive 70- $\mu \mathrm{m}$ quiet clumps with several surveys of massive dense cores at different evolutionary phases (Traficante et al. 2018). We showed that the three Larson relations seem to be violated in massive starforming regions at the scales of clumps and cores. However, a consistent analysis on a large sample of hundreds of massive starforming clumps at various evolutionary stages has not yet been performed.

In this work, we examine the three Larson relations and their implications in a large sample of massive clumps obtained from a combination of the Elia et al. (2017) catalogue of clumps extracted from the Herschel infrared Galactic Plane (Hi-GAL) survey (Molinari et al. 2010), with a sample of molecular lines observed at $3 \mathrm{~mm}$ with the Millimetre Astronomy Legacy Team $90 \mathrm{GHz}$ (MALT90) survey (Jackson et al. 2013). In Section 2, we describe the data sets used in this work and the selection of the final sample of 213 clumps with well-defined dust and gas emission properties. In Section 3, we describe the classification scheme adopted for these clumps. In Section 4, we explore in detail the three Larson relations and we discuss the validity of these relations in massive clumps at different evolutionary stages. In Section 5, we analyse the implications of the previous results, in particular in the interpretation of the virial parameter. In Section 6, we study the properties of the clumps that show signs of infall motions and we compare these results with the rest of the sample. In Section 7, we explore possible explanations for the observed non-thermal motions. Finally, in Section 8 we draw our conclusions.

\section{DATA SETS AND CLUMP SELECTION}

In the following, we describe the main data sets that we have considered in this work, the selection criteria used to obtain the final sample and the estimation of the uncertainties on the main parameters used in the rest of the paper.

\subsection{Hi-GAL data}

The Hi-GAL survey observed the whole Galactic plane in five wavelengths $(70,160,250,350$ and $500 \mu \mathrm{m})$ using two instruments: the photodetector array camera and spectrometer (PACS; 70 and $160 \mu \mathrm{m}$; Poglitsch et al. 2010) and the spectral and photometric imaging receiver (SPIRE; 250, 350 and $500 \mu \mathrm{m}$; Griffin et al. 2008). This survey identified tens of thousands of filaments (Schisano et al. 2014) and point sources (Molinari et al. 2016b) across the Galaxy. The band-merged catalogue contains $\simeq 100000$ sources in the longitude range $-71^{\circ} \leq l \leq 67^{\circ}$ with defined spectral energy distributions (SEDs) and clump properties (Elia et al. 2017), from which we extracted the clumps used in this work.

\subsubsection{Complementary dust continuum data sets}

The Hi-GAL fluxes have been complemented at longer wavelengths with data at $870 \mu \mathrm{m}$ taken from the APEX Telescope Large Area Survey of the Galaxy (ATLASGAL) survey (Schuller et al. 2009). This survey covers the Galactic longitudes $-80^{\circ} \leq l \leq 60^{\circ}$ with a spatial resolution of 19.2 arcsec and a sensitivity of $\simeq 70 \mathrm{mJy}$ beam $^{-1}$ (Csengeri et al. 2014). The ATLASGAL clumps catalogue (Csengeri et al. 2014) contains $\simeq 10000$ sources, including all the sources presented in this work.

The far-infrared (FIR) submm fluxes have also been complemented at shorter wavelengths with mid-infrared (MIR) data at 21 $\mu \mathrm{m}$ from the Midcourse Space Experiment (MSX; Egan, Price \& Kraemer 2003), at $22 \mu \mathrm{m}$ from the Wide-field Infrared Survey Explorer (WISE; Wright et al. 2010) and at $24 \mu \mathrm{m}$ from the survey of the Galactic plane using the multiband imaging photometer for Spitzer (MIPSGAL; Gutermuth \& Heyer 2015). The MIR counterparts are described in the Elia et al. (2017) catalogue. We also used the results of the Red MSX Source (RMS) survey (Lumsden et al. 2013) to classify the clumps. The RMS survey is a midinfared (MIR) selection of massive, evolved young stellar object (YSO) candidates across the whole Galaxy identified by the MSX. Details of the survey can be found in Lumsden et al. (2013). The source counterparts at 21,22 and $24 \mu \mathrm{m}$ have been used to determine the evolutionary sequence of the clumps according to the scheme proposed in Merello et al. (in preparation) and summarized in Section 3.

\subsection{MALT90 data}

The MALT90 survey (Jackson et al. 2013) is a large survey of 90$\mathrm{GHz}(\simeq 3-\mathrm{mm})$ emission lines associated with star-forming regions. The survey observed 2012 clumps chosen from the ATLASGAL survey (Schuller et al. 2009). The clumps are distributed in the Galactic longitude ranges $3^{\circ} \leq l \leq 20^{\circ}$ in the first quadrant and $300^{\circ} \leq l \leq 357^{\circ}$ in the fourth quadrant. The survey has been carried out with the 22-m Mopra telescope in on-the-fly mapping mode, covering a region of $3.4 \times 3.4 \mathrm{arcmin}^{2}$ across each clump, centred in the ATLASGAL clump centroid position. The FWHM is 38 arcsec at $90 \mathrm{GHz}$ and the velocity resolution is $0.11 \mathrm{~km} \mathrm{~s}^{-1}$. Typical system temperatures were in the range $180 \leq T_{\text {sys }} \leq 300 \mathrm{~K}$, for a typical rms noise of $\simeq 250 \mathrm{mK}$ per channel (Jackson et al. 2013). The MALT90 survey observed 16 different species spanning from dense gas tracers of relatively quiescent gas, such as $\mathrm{N}_{2} \mathrm{H}^{+}$(1-0), up to shock tracers, such as $\mathrm{SiO}(1-0)$, and ionized gas tracers, such as H41 $\alpha$ (Jackson et al. 2013). 


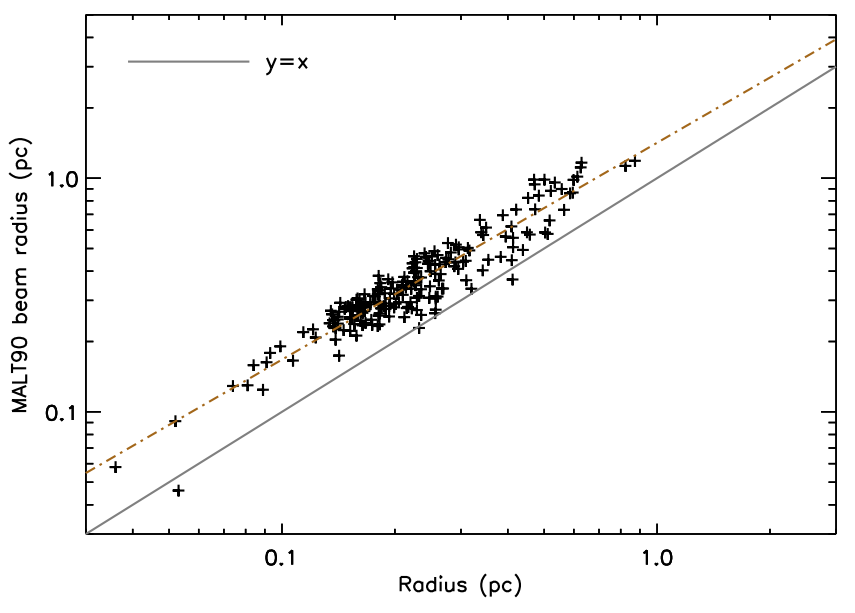

Figure 1. Comparison between the radius of clumps estimated at $250 \mu \mathrm{m}$ and the radius of a region equal to the size of a MALT90 beam. There is a strong correlation between the two values, and a systematic offset, which shows that the MALT90 beam region is always larger than the size of the Hi-GAL clump. The grey line is the $y=x$ line. The red-dashed line is the fit of the distribution. The offset between the two lines has been used to estimate the filling factor, which is 0.64 .

\subsection{Clump selection}

We combined the data sets provided by the Hi-GAL and MALT90 surveys to identify a statistically significant sample of clumps with known distances and well-defined dust and emission-line properties.

From the 2012 Hi-GAL clumps also observed in the MALT90 survey, we first excluded all clumps with longitudes $l \leq\left|10^{\circ}\right|$, for which the distance estimation might be highly inaccurate. We also excluded all the clumps with a mass estimation $M \leq 5 \times \sigma_{\mathrm{err}}$, where $\sigma_{\text {err }}$ is the error associated with the mass estimation, as discussed by Elia et al. (2017). We obtain a first selection of 617 clumps.

We further restricted our sample to well-defined $\mathrm{N}_{2} \mathrm{H}^{+}$ (1-0) spectra that we used to estimate the gas velocity dispersion. The $\mathrm{N}_{2} \mathrm{H}^{+}(1-0)$ emission of each clump was evaluated from the MALT90 data cubes by averaging the spectrum across all the pixels within one MALT90 beam, $\simeq 38$ arcsec. We assumed that all the $\mathrm{N}_{2} \mathrm{H}^{+}$emission comes from the clumps, and we estimate the filling factor from the comparison of the radius of each Hi-GAL clump with the radius of a region equal to the MALT90 beam (Fig. 1). There is a strong correlation between these two quantities, and the size of the Hi-GAL clumps is systematically smaller than the radius estimated from the MALT90 beam for a factor of 0.64 , on average. We assumed an average filling factor of 0.64 for the entire sample. The MALT90 data cubes are given in antenna temperature $T_{\mathrm{A}}^{*}$ and they have been converted to the main beam temperature $T_{\mathrm{MB}}=T_{\mathrm{A}}^{*} / \eta_{\mathrm{MB}}$, assuming a mean beam efficiency $\eta_{\mathrm{MB}}=0.49$ (Miettinen 2014). The properties of each $\mathrm{N}_{2} \mathrm{H}^{+}(1-0)$ averaged spectrum have been extracted in IDL using a hyperfine fitting routine and the mp $\mathrm{i} t \mathrm{fun}$ algorithm (Markwardt 2009), after smoothing the data to a spectral resolution of $0.3 \mathrm{~km} \mathrm{~s}^{-1}$ to enhance the signal-to-noise $(\mathrm{S} / \mathrm{N})$ ratio. We excluded all clumps with a $\mathrm{S} / \mathrm{N}$ ratio below 5 , where the rms in each smoothed data cube has been measured in a $100 \mathrm{~km} \mathrm{~s}^{-1}$ wide spectral window near the $\mathrm{N}_{2} \mathrm{H}^{+}$emission. We further excluded clumps for which the fit converged but the spectrum was affected by spikes and/or by multiple components along the line of sight. Using these criteria, we obtained 308 clumps. We completed our selection by excluding all clumps without a clear distance assignation, in particular without a well-defined resolution of the near-far distance ambiguity. First, we have refined the kinematic distances in the Elia et al. (2017) catalogue (and the quantities that depend on them) with the newest set of distances developed for the Hi-GAL survey under the VIALACTEA project (Mege et al., in preparation). The method used by Elia et al. (2017) was the same as in Russeil et al. (2011): the brightest emission lines in the ${ }^{12} \mathrm{CO}$ or ${ }^{13} \mathrm{CO}$ spectra along the line of sight of each source are used to estimate the velocities of the local standard of rest and converted into heliocentric distances using the Brand \& Blitz (1993) rotation curve. The distances in Mege et al. (in preparation) have been determined using a similar approach, but including all the recent surveys of the Galactic plane to trace structures along the line of sight, and using the more recent Reid et al. (2009) rotation curve. Then, in order to identify only clumps with a well-defined distance estimation, we have compared the distances assigned to our 308 clumps with the distances of the MALT90 sample estimated in Whitaker et al. (2017) and of the ATLASGAL sources published in Urquhart et al. (2018). We excluded from the sample all sources with a difference in the distance estimation larger than 20 per cent among the three surveys.

We obtain a final selection of 213 clumps with well-defined distances, dust properties and $\mathrm{N}_{2} \mathrm{H}^{+}$spectra. The properties of these clumps are summarized in Appendix B.

\subsection{Estimation of uncertainties}

In this section, we analyse the main source of uncertainties in both dust and gas properties of our 213 clumps. The results discussed in the rest of the work following subsections are significantly affected by the statistical uncertainties associated with each parameter, while they are not affected by uncertainties that produce systematic offsets. The dust properties are mainly affected by the following sources of uncertainties.

\section{Dust models for cold dust}

The dust properties of the clumps in the Elia et al. (2017) catalogue have been evaluated assuming a single-temperature greybody model with a spectral index $\beta=2.0$, an opacity $\kappa_{0}=0.1$ at $\lambda_{0}=300 \mu \mathrm{m}$ (Beckwith et al. 1990) and a gas-to-dust ratio of 100. The commonly used model of Ossenkopf \& Henning (1994), assuming a thin ice mantle and a gas density of $10^{6} \mathrm{~g} \mathrm{~cm}^{-3}$ leads to $\kappa_{0}=0.17$ at $\lambda_{0}=300 \mu \mathrm{m}$, a difference of almost a factor of 2 from the Beckwith et al. (1990) opacity. Because the mass estimate scales linearly with the opacity, the use of a different model would lead to a systematic offset in the mass, surface density (positive offset for higher values of $\kappa_{0}$ ) and virial parameter estimates (negative offset). However, our results are not affected by systematic offsets. The spectral index $\beta$ can vary across different sources as a function of the dust temperature (Paradis et al. 2010), and as a function of the dust column density (Juvela et al. 2015). With a variation of the spectral index $\beta=2.0 \pm 0.3$ (in line with the findings of Paradis et al. 2010), the mass changes accordingly for a factor of $\simeq 30$ per cent. We consider this value as the uncertainty on the mass estimation due to the assumed dust model.

\section{Errors in distance estimation}

These uncertainties are a result of the following.

(i) The method used to estimate the radial velocities. The distances presented in the catalogue of Mege et al. (in preparation) are evaluated using a method similar to that adopted by Urquhart et al. 
(2018), who calculated an uncertainty on the distance estimation of $\simeq 0.30 \mathrm{kpc}$. Our final sample of 213 sources is at a mean distance of $\simeq 4.2 \mathrm{kpc}$, which gives an error of $\simeq 7$ per cent on the distance estimation due to the adopted method.

(ii) The rotation curve adopted to convert the radial velocities into kinematic distances. Russeil et al. (2011) compared the distance obtained using the rotation curves of Brand \& Blitz (1993) and Reid et al. (2009) and they showed that, within the uncertainties, the results are compatible. Therefore, the results are not greatly affected by different rotation curves.

(iii) The near-far distance ambiguity. As discussed in Section 2.3, we have selected only the conservative, but most reliable subsample of sources with the same solution for the near-far distance ambiguity in the Hi-GAL (Mege et al., in preparation), ATLASGAL (Urquhart et al. 2018) and MALT90 (Whitaker et al. 2017) catalogues. Therefore, we have assumed that the distance ambiguity has been solved for our subsample of sources.

These sources have a distance determination that differs up to 20 per cent with respect to the values in the ATLASGAL and MALT90 catalogues, but on average the difference is only 4 per cent. Combining these results, we conservatively assume that the distance uncertainties are of the order of 15 per cent. The same uncertainties are associated with the radius $R$. The mass depend on distance as $M \propto d^{2}$, so the mass uncertainties due to the distance uncertainties are $\simeq 30$ per cent.

\section{Uncertainties on radius estimation}

The angular radius of each clump is defined as the geometrical mean $R_{\text {eq }}$ of the two FWHMs of the Gaussian fit carried out at $250 \mu \mathrm{m}$ (Elia et al. 2017). However, the majority of these sources are elongated along one direction, and this asymmetry produces uncertainties in the definition of the source radius. We estimated these uncertainties by taking the differences between $R_{\text {eq }}$ and the minor and major axis of each source. The average differences are of the order of 10 per cent of the geometrical mean, with peaks of up to 50 per cent and a standard deviation of $\simeq 10$ per cent. We consider a conservative value of 20 per cent on the uncertainties associated with the radius estimation due to the geometrical mean approximation.

\section{Results of the SED fitting routine}

Mass and temperature are estimated only for clumps that have at least three consecutive fluxes in the Hi-GAL wavelengths $160 \leq$ $\lambda \leq 500 \mu \mathrm{m}$, and irregular SEDs are not considered (Elia et al. 2017). The clumps in our sample have well-defined properties and the uncertainties associated with the fitting routine are very small. They are of the order of 1.5 per cent, with a peak of 18 per cent. We assume an average error of 5 per cent associated with the SED fitting.

\section{Choice of the photometry algorithm}

In Appendix A, we discuss how the estimates of properties such as the mass differ from the values of the Elia et al. (2017) catalogue using a different algorithm to evaluate the source photometry (Hyper; Traficante et al. 2015b). The differences in the estimation of the fluxes produce a systematic offset of 10 per cent in the mass values, which do not bias the results of this work. The statistical uncertainties are of the order of $\simeq 25$ per cent, which we assume to be the uncertainties on the mass estimation due to the photometry method. In Appendix A, we also show that our results are robust to
Table 1. Relative uncertainties associated with the main parameters used in this work. The relative uncertainties are estimated from the discussion in Section 2.4. The sources of uncertainties are used to estimate the relative uncertainties on the parameters.

\begin{tabular}{|c|c|c|}
\hline Parameter & $\begin{array}{c}\text { Relative } \\
\text { uncertainties }\end{array}$ & Source of uncertainties \\
\hline$R$ & $25 \%$ & Distance, geometrical mean \\
\hline$M$ & $50 \%$ & $\begin{array}{l}\beta \text { index, distance, SED fitting, } \\
\text { photometry method }\end{array}$ \\
\hline$\Sigma$ & $35 \%^{a}$ & $\begin{array}{l}\beta \text { index, SED fitting, } \\
\text { photometry method }\end{array}$ \\
\hline$\sigma$ & $30 \%$ & $\begin{array}{l}\text { Hyperfine fitting, } \\
\text { thermal motions }\end{array}$ \\
\hline$\alpha_{\mathrm{vir}}$ & $65 \%$ & $M, R, \sigma$ \\
\hline
\end{tabular}

$\overline{a^{a}}$ The uncertainties on $\Sigma$ depend only on the SED fitting and are independent of the source distance.

these differences, and they are not biased by the specific algorithm used to extract the properties of the clumps.

\section{Uncertainties on velocity dispersion estimation}

The uncertainties associated with the estimation of the non-thermal velocity dispersion are dominated by the spectral resolution of our observations. The hyperfine fitting has been carried out on spectra smoothed three times, and the uncertainties on the fit are of the order of the smoothed spectral resolution, $0.3 \mathrm{~km} \mathrm{~s}^{-1}$. The average non-thermal component of the velocity dispersion is $\simeq 1.21 \mathrm{~km} \mathrm{~s}^{-1}$, so the error derived from the hyperfine fit is of the order of 25 per cent of the measured non-thermal velocity dispersion.

The intensity of the thermal component to be subtracted from the observed velocity dispersion could be another source of uncertainty. We estimated this component assuming the same temperature for the gas as for the clump, which spans a range $8.5 \leq T \leq 40 \mathrm{~K}$. The $\mathrm{N}_{2} \mathrm{H}^{+}$thermal component within this range of temperatures is much smaller than the non-thermal component, and varies in the range $0.05 \leq \sigma_{\text {th }} \leq 0.11 \mathrm{~km} \mathrm{~s}^{-1}$. Even accounting for a gas temperature that differs substantially from the estimated dust temperature, the error is $\sigma_{\text {th, unc }} \lesssim 0.05 \mathrm{~km} \mathrm{~s}^{-1}$. This is smaller than 5 per cent of the measured velocity dispersion. We assume a conservative error of 5 per cent from the subtraction of the thermal component to the estimation of the non-thermal motions.

The uncertainties on the main parameters used in this work are summarized in Table 1 . The uncertainties on $\alpha_{\text {vir }}$ have been evaluated using the standard formula for the propagation of uncertainties.

\section{CLUMP CLASSIFICATION SCHEME}

The association between our $213 \mathrm{Hi}-\mathrm{GAL}$ clumps and their counterparts in the MIPSGAL, WISE and RMS surveys are used to determine an evolutionary sequence for our sources. The association with MIPSGAL and WISE has been taken from the Elia et al. (2017) catalogue. The association with RMS has been made by looking for RMS counterparts of the Hi-GAL sample within a radius equal to the geometrical mean of the FWHMs of each source.

We adopted the evolutionary scenario based on the Hi-GAL survey and presented in Merello et al. (in preparation). These authors analysed $\simeq 1000 \mathrm{Hi}$-GAL clumps and followed a similar approach to that used by König et al. (2017), although they divided the clumps into five different evolutionary phases, as follows.

(i) A starless phase, which is identified as a bright region at wavelengths $\lambda \geq 160 \mu \mathrm{m}$ but still dark at wavelengths $\lambda<100 \mu \mathrm{m}$. 
Table 2. Classification scheme of our 213 clumps following the evolutionary scenario described in Merello et al. (in preparation). In column 2, we give the wavelengths at which each evolutionary stage becomes bright. In column 3, we list the surveys that have a visible counterpart in the data. Column 4 shows the median value of $L / M$ and column 5 gives the number of identified objects.

\begin{tabular}{|c|c|c|c|c|}
\hline $\begin{array}{l}\text { Evolutionary } \\
\text { phase }\end{array}$ & $\begin{array}{c}\text { Bright } \\
\text { wavelengths }(\mu \mathrm{m})\end{array}$ & Survey & $\begin{array}{c}L / M \\
\left(\mathrm{~L}_{\odot} / \mathrm{M}_{\odot}\right)\end{array}$ & Count \\
\hline Starless & $>70$ & Hi-GAL & 1.7 & 14 \\
\hline Protostellar MIR bright & $\geq 21$ & Hi-GAL, MIPSGAL, WISE & 6.4 & 106 \\
\hline YSOs & $\geq 8$ & Hi-GAL, MIPSGAL, WISE, RMS & 32.1 & 25 \\
\hline $\mathrm{H}_{\mathrm{II}}$ regions $\left(\mathrm{UCH}_{\mathrm{II}}+\right.$ ext. $\left.\mathrm{H}_{\mathrm{II}}\right)$ & $\geq 8$ and radio emission & Hi-GAL, MIPSGAL, WISE, RMS & 36.3 & $56(42+14)$ \\
\hline
\end{tabular}

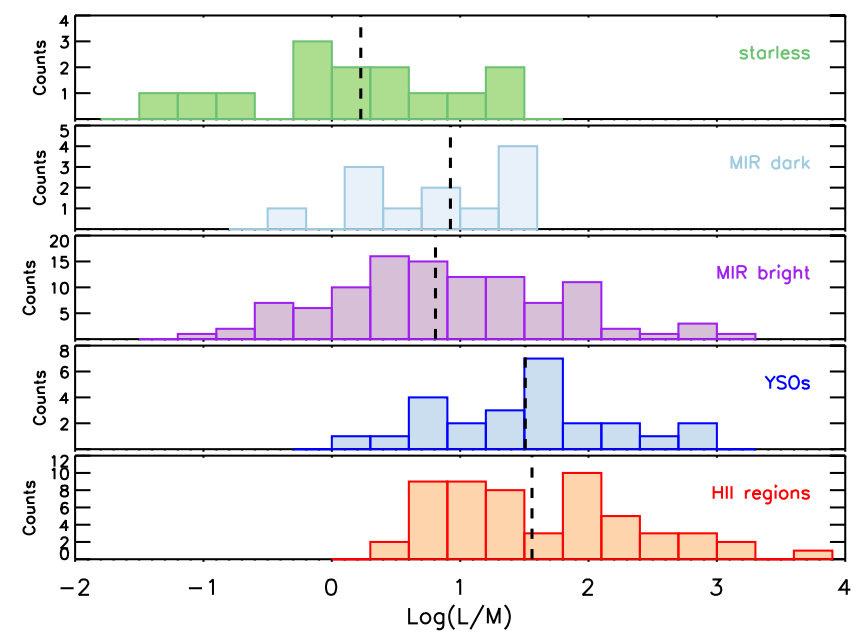

Figure 2. $L / M$ ratio distribution of our 213 clumps divided into the various evolutionary phases. The entire sample spans more than four orders of magnitude and there is an evident increase of the $L / M$ ratio going from starless to $\mathrm{H}_{\text {II }}$ regions.

(ii) Protostar MIR dark, which is when a clump becomes visible at $70 \mu \mathrm{m}$ but it is still dark in the MIR, or the emission is too faint to be identified. These clumps are bright at all Hi-GAL wavelengths, with no counterparts in the MIPSGAL, WISE and RMS surveys.

(iii) Protostar MIR bright, which is when the clumps also become visible in the MIR and their bolometric luminosity increases significantly. These clumps have at least one counterpart in one of the MIPSGAL, WISE and MSX surveys but they do not pass the RMS criteria to be classified as YSOs (Lumsden et al. 2013).

(iv) YSOs, which is when the protostars have reached the zeroage main sequence and have also become bright in the NIR regime. They are classified as YSOs in the RMS survey.

(v) $\mathrm{H}_{\text {II }}$ regions, where the thermal bremsstrahlung emission of the gas ionized in the envelope of the more massive stars can be observed at radio wavelengths (Wood \& Churchwell 1989). Radio observations are used to identify $\mathrm{H}_{\mathrm{II}}$ regions among YSOs (Hoare et al. 2007). These sources have been classified as either UCH II or extended $\mathrm{H}$ II regions in the RMS catalogue.

Based on this classification scheme, which is summarized in Table 2, we have identified: 14 starless clumps, 12 protostar MIR dark clumps, 106 protostar MIR bright clumps, 25 YSOs and $56 \mathrm{H}$ II regions, among which 14 are extended $\mathrm{H}$ II regions. This classification is consistent with the results we obtain from a well-known indicator of clump evolution, the luminosity over mass $(L / M)$ ratio (Molinari et al. 2008; Molinari et al. 2016a). As shown in Fig. 2, the $L / M$ ratio of these clumps spans more than four orders of magnitude in total, and increases from starless to $\mathrm{H}$ II regions. In agreement with

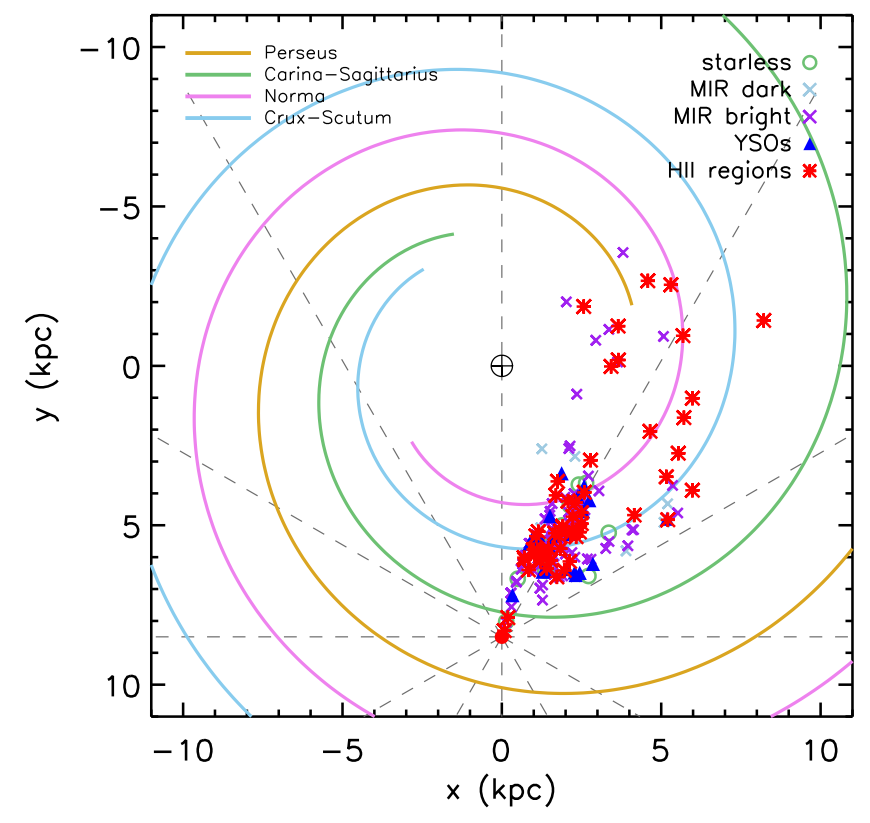

Figure 3. Galactic distribution of the 213 clumps investigated in this work. Colours and symbols represent clumps at different evolutionary stages. Overlaid is the Galactic model of Hou et al. (2009), the same model used to discuss the clump distribution in Elia et al. (2017). All clumps are in the IV Quadrant, mostly distributed across the Crux-Scutum and Norma arms and in the inter-arm region. The red-filled dot at $[0,8.5] \mathrm{kpc}$ represents the position of the Sun. The black circle at $[0,0] \mathrm{kpc}$ is the Galactic Centre.

the findings of Merello et al. (in preparation), there is no significant difference in $L / M$ between MIR dark and MIR bright sources, suggesting that the presence of a MIR source in a clump does not significantly alter the total luminosity of the cold dust envelope.

In Fig. 3, we present the Galactic distribution of our sources, overlaid with the four spiral-arm Galactic model of Hou, Han \& Shi (2009). All sources are located in the IV Quadrant. They are mostly concentrated in a region between the Crux-Scutum and the Norma arms, and the inter-arm region.

In the next Sections, we investigate the gravo-turbulent properties of these clumps, in light of this classification scheme.

\section{LARSON RELATIONS IN MASSIVE CLUMPS}

In this section, we analyse the three Larson relations in our clumps and we discuss the implications of the results. 


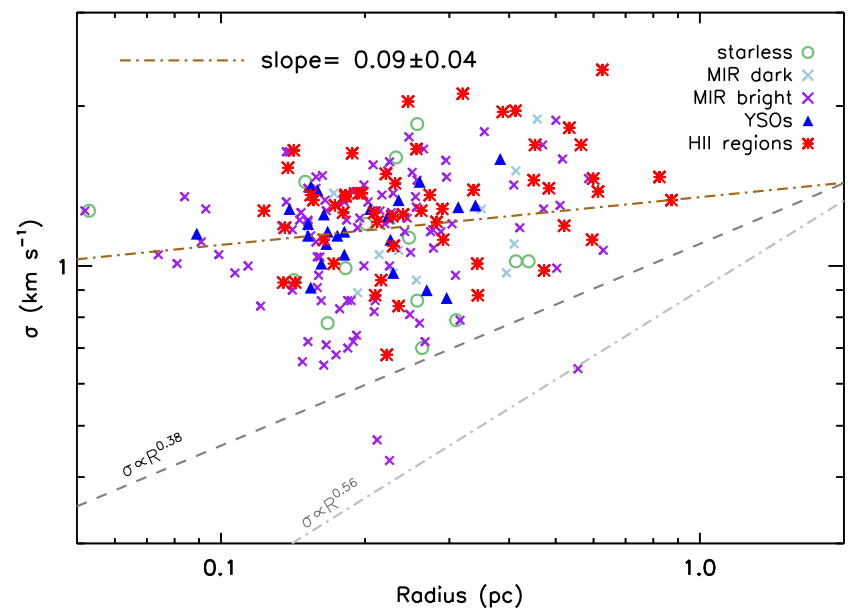

Figure 4. First Larson relation: velocity dispersion $\sigma$ as a function of radius $R$. The dark grey dashed line is the original Larson relation, $\sigma \propto R^{0.38}$, and the light grey dash-dotted line is the revised Heyer \& Brunt (2004) relation, $\sigma \propto R^{0.56}$. The correlation is weak, with a Pearson coefficient of $\rho=0.26$.

\subsection{The first Larson relation: linewidth-size}

The first Larson relation shows the proportionality between the size of GMCs and the non-thermal motions of the gas in the region (Larson 1981). This relation has often been considered to be the result of interstellar turbulence. The interstellar medium modelled as a turbulent fluid dominated by shocks follows a power-spectrum relation of the form $R \propto \sigma^{0.5}$ (i.e. a Burgers-like power spectrum $E_{k} \propto k^{-2}$; e.g. McKee \& Ostriker 2007), a scenario that reproduces large-scale observations (Padoan \& Nordlund 2002; McKee $\&$ Ostriker 2007). However, the relation seems to break in massive clumps embedded in molecular clouds. For example, Caselli \& Myers (1995) observed the Orion A and B high-mass star-forming regions and they found a correlation between size and velocity dispersion of the form $R \propto \sigma^{0.21}$, significantly lower than that found in GMCs. Similar results have been obtained in the survey of highmass star-forming regions of Shirley et al. (2003). At the same time, other surveys of massive star-forming objects found no correlation (Plume et al. 1997; Ballesteros-Paredes et al. 2011; Traficante et al. 2018) or even an inverse correlation (Wu et al. 2010) between size and linewidth.

In Fig. 4, we report the velocity dispersion-radius relation for our sample of 213 sources. The slope of the linear fit in the log-log space is $0.09 \pm 0.04$, suggesting that a correlation between velocity dispersion and radius, if present, is very low. The fit in this plot (and for the rest of this work) has been obtained from a linear regression carried out with the fitexy IDL routine, which performs a $\chi^{2}$ approximation when uncertainties are known in both the $x$ and $y$ variables. The Pearson correlation coefficient $\rho$ measures the linear correlation between the two variables and varies in the range $-1 \leq \rho$ $\leq 1$, where $\rho=-1$ indicates total anticorrelation, $\rho=1$ indicates total correlation and $\rho=0$ indicates no correlation. Note that $\rho$ $\simeq 0.26$, which suggests that the correlation between the velocity dispersion and radius is weak.

In Fig. 5, we report the quantity $\sigma / R^{0.5}$ divided for the different evolutionary stages. Following the first Larson relation, this quantity should be a constant of the system. Instead, we find a distribution of this quantity across a range $0.9 \leq \sigma / R^{0.5} \leq 12.8 \mathrm{~km} \mathrm{~s}^{-1} \mathrm{pc}^{-1 / 2}$, which is larger than the estimated uncertainties of size and velocity dispersion combined (see Section 2.4). Also, within uncertainties, there is no distinction between different evolutionary phases, with

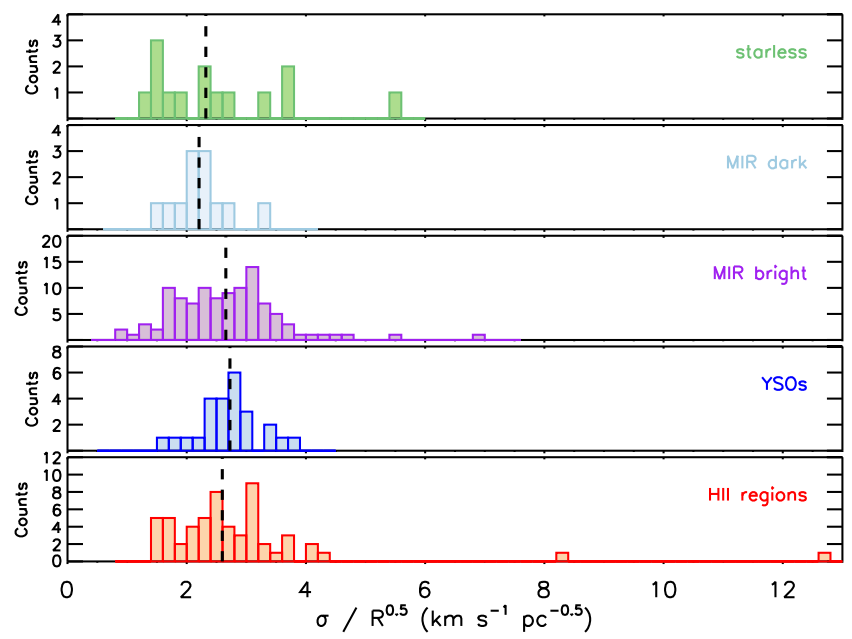

Figure 5. The first Larson relation distribution for our clumps, divided for different evolutionary stages. The relation states that the quantity $\sigma / R^{0.5}$ should be a constant. Instead, the distributions span a range of values for each evolutionary phase.

median values of $[2.32,2.21,2.66,2.73,2.60] \mathrm{km} \mathrm{s}^{-1} \mathrm{pc}^{-0.5}$ in the starless, protostar MIR dark, protostar MIR bright, YSOs and $\mathrm{H}_{\mathrm{II}}$ regions evolutionary phases, respectively. Altogether or divided for different evolutionary phases, these results suggest that the first Larson relation typically breaks down at clump scales, and this break is not because of the different internal conditions of these objects.

The observed first Larson relation implies that one, or both, of the other two relations must not be followed by this ensemble of clumps.

\subsection{The second Larson relation: the virial equilibrium}

The second Larson relation states that GMCs are approximately in virial equilibrium. The virial parameter $\alpha_{\text {vir }}$ has often been interpreted as representative of the equilibrium between $E_{k}$ and $E_{G}$ when all other forces such as magnetic fields are not involved (and assuming spherical and homogeneous density distribution; Bertoldi $\&$ McKee 1992). The virial equilibrium implies $\alpha_{\mathrm{vir}}=\alpha_{\mathrm{eq}}=1$ or, if a collapsing cloud is modelled as an isothermal (Bonnor-Ebert) sphere, the hydrostatic equilibrium is at $\alpha_{\text {eq }} \simeq 2$ (Kauffmann, Pillai \& Goldsmith 2013; Tan et al. 2014). GMCs are expected to be in virial equilibrium, with the kinetic energy due to local turbulence that provides support against the gravitational collapse (McKee \& Tan 2003; Heyer et al. 2009). The formation of massive clumps in a gravo-turbulent collapse is also predicted to occur in a state of global virial equilibrium (Lee \& Hennebelle 2016).

Alternatively, the observed non-thermal motions might be partly the result of the collapse itself, and might not necessarily provide support against gravity. In this interpretation, virial equilibrium loses its original meaning. The regions would be in approximately virial equipartition (which also implies $\alpha_{\mathrm{eq}}=2$ ), but misinterpreted as in virial equilibrium (Ballesteros-Paredes 2006).

Independently of the interpretation of the observed $\alpha_{\text {vir }}$, there is a general consensus that regions with $\alpha_{\text {vir }}<\alpha_{\text {eq }}$ are gravitationally bound and prone to collapse, if not sustained by strong magnetic fields that can stabilize them (e.g. Kauffmann et al. 2013). These regions do not follow the second Larson relation.

Fig. 6 shows the distribution of the virial parameter of our 213 clumps divided into the five evolutionary stages, and in Table 3 we report the range of $\alpha_{\text {vir }}$ for each phase. The virial parameter 


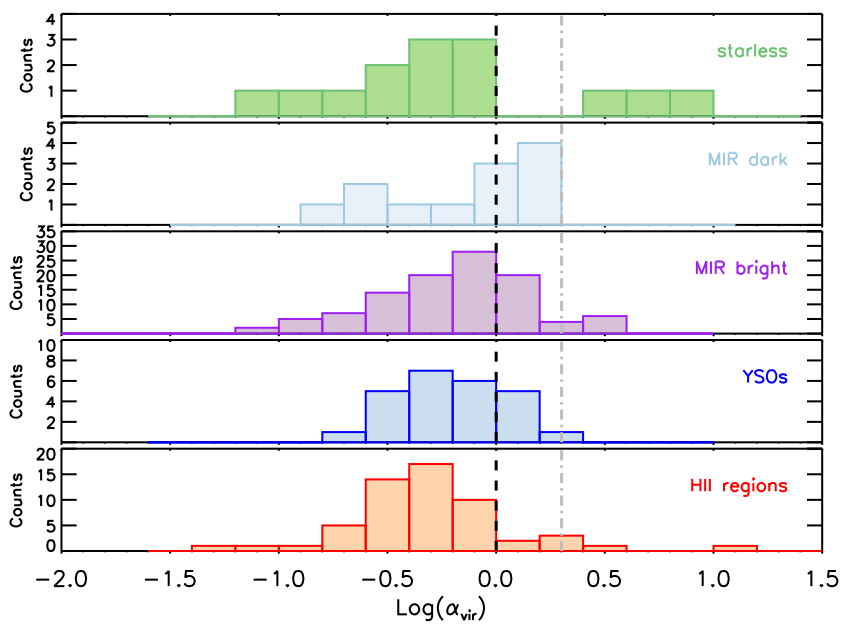

Figure 6. Histogram of the $\alpha_{\text {vir }}$ distribution at different evolutionary stages. The majority of the clumps have $\alpha_{\text {vir }} \leq 2$, independent of the evolutionary phase. The black dashed line corresponds to $\alpha_{\mathrm{vir}}=1$ and the grey dashdotted line corresponds to $\alpha_{\mathrm{vir}}=2$.

Table 3. Range of values of $\alpha_{\text {vir }}$ in our clumps for each stage of evolution.

\begin{tabular}{lc}
\hline Evolutionary phase & $\alpha_{\text {vir }}$ \\
\hline Starless & $0.07 \leq \alpha_{\text {vir }} \leq 7.98$ \\
MIR dark & $0.13 \leq \alpha_{\text {vir }} \leq 1.71$ \\
MIR bright & $0.07 \leq \alpha_{\text {vir }} \leq 3.52$ \\
YSOs & $0.20 \leq \alpha_{\text {vir }} \leq 2.03$ \\
H II regions & $0.05 \leq \alpha_{\text {vir }} \leq 12.81$ \\
\hline
\end{tabular}

spans the range $0.05 \leq \alpha_{\text {vir }} \leq 12.8$, and each evolutionary phase spans at least one order of magnitude, with no clear differences between the various stages of evolution. A total of 51 clumps have $\alpha_{\text {vir }} \geq 1$, and only 14 have $\alpha_{\text {vir }} \geq 2$. The majority of our clumps are gravitationally bound and these clumps, if not sustained by strong magnetic fields (see Section 7.3), are not in gravitational equilibrium. If the kinetic energy is due to turbulence acting to support gravity, then its contribution is not sufficient to stop or slow down the collapse at the clump scales.

\subsection{The third Larson relation: mass-radius diagram}

A practical form of the third Larson relation states that molecular clouds have approximately the same surface density: $\Sigma \propto n R \propto$ $R^{-0.1}$. This formulation is much easier to verify experimentally, as it does not require placing any constraints on the third dimension needed to evaluate the volume density of the observed regions.

The early observations of Larson (1981) suggest that GMCs all have similar column densities. However, the third Larson relation might simply be an observational bias due to the molecular tracer used in early GMC observations (e.g. Kegel 1989; BallesterosParedes 2006; Heyer et al. 2009). Using extinction as a tracer of molecular gas, Lombardi, Alves \& Lada (2010) demonstrated that the third Larson relation is observed in nearby molecular clouds only above a given surface density threshold. The relation does not hold in clumps and cores embedded in single clouds, and an apparent density-size relation can be observed as an artefact of clumps limited within column density thresholds (Camacho et al. 2016; Ballesteros-Paredes et al. 2012). Indeed, several surveys of

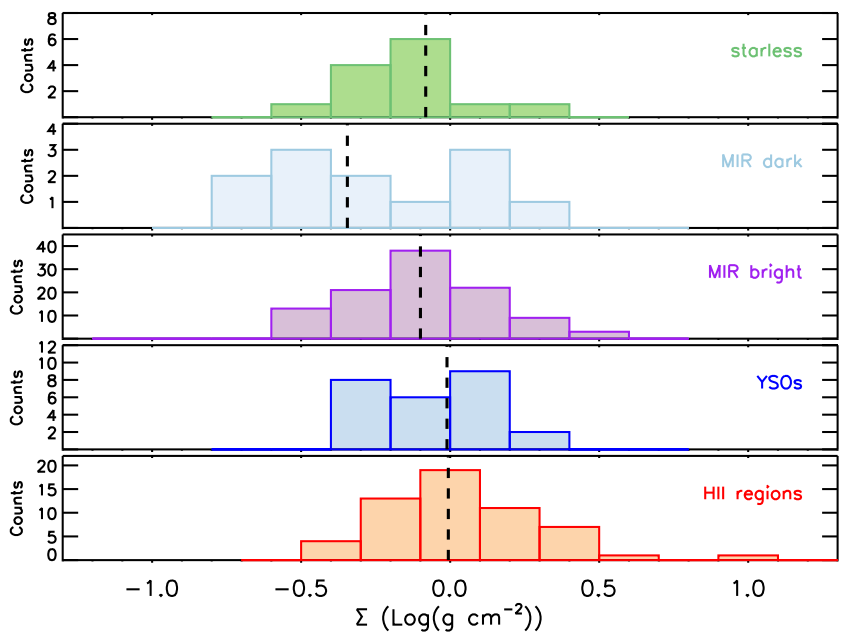

Figure 7. Surface density distribution of the clumps separated for each evolutionary phase. The black vertical lines correspond to the median values of $\Sigma$ in each evolutionary stage. The surface density values span more than one order of magnitude, and there is no clear evidence of a trend with evolution.

massive clumps have shown that they span almost two orders of magnitude in surface densities (Urquhart et al. 2014; Traficante et al. 2015a; Svoboda et al. 2016; Elia et al. 2017).

The surface densities of the 213 clumps analysed in this work are shown in Fig. 7. The surface densities are in the range $0.13 \leq \Sigma \leq$ $8.57 \mathrm{~g} \mathrm{~cm}^{-2}$, spanning more than one order of magnitude in each evolutionary phase. We found median values of [0.83, 0.45, 0.80, $0.98,0.99] \mathrm{g} \mathrm{cm}^{-2}$ in starless, protostar 24- $\mu \mathrm{m}$ dark, protostar 24$\mu \mathrm{m}$ bright, YSOs and $\mathrm{H}$ II regions evolutionary phases, respectively. Within the uncertainties in the estimation of the surface densities (35 per cent of their value; Table 1), the median distributions are likely indistinguishable, in agreement with the findings of Urquhart et al. (2014) and Svoboda et al. (2016), and with the results presented in Merello et al. (in preparation). The surface density of each clump is more likely to be related to the density properties of the local environment, regardless of its evolution.

\section{Mass-radius relation}

An alternative way to look at the third Larson relation is through the mass-radius diagram. A sample of star-forming regions with roughly constant column density should have a mass distribution $M$ $\propto R^{\delta}$ with $\delta \simeq 2$. Previous surveys of massive clumps have found a large range of values for $\delta$, which is strongly dependent on the different strategies used to extract the dust properties of the clumps. Mass-radius diagrams have been observed with slopes in the range $\delta \simeq 1.6-1.7$ (Lombardi et al. 2010; Kauffmann et al. 2010; Urquhart et al. 2014), as well as with $\delta \geq 2$ (Ellsworth-Bowers et al. 2015) or even greater ( $\delta \geq 2.7$; Ragan, Bergin \& Gutermuth 2009).

In Fig. 8 , we show the mass-radius diagram of our clumps. The fit has a slope $\delta=2.38 \pm 0.10$, not in agreement with constant $\Sigma$. We investigated how much this result is sensitive to the estimated uncertainties by modifying the errors associated with the parameters of \pm 10 per cent. We obtained a difference of up to 5 per cent in the value of the slope, which varies in the range $2.19 \pm 0.09 \leq \delta \leq$ $2.53 \pm 0.13$. Also accounting for these variations, the slope is not consistent with constant surface density, as expected from the large spread shown in Fig. 7. 


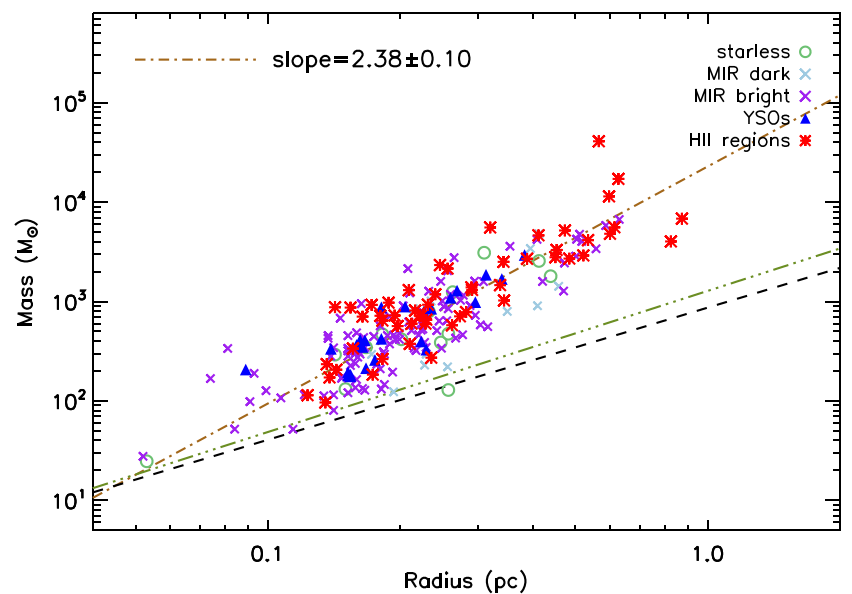

Figure 8. Mass-radius distribution. The line black dashed line is the Kauffmann \& Pillai (2010) massive star formation threshold, $M(r)>870 \mathrm{M}_{\odot}(R / \mathrm{pc})^{1.33}$, and the green dash-dotted line is the revised Baldeschi et al. (2017) threshold, $M(r)>1282 \mathrm{M}_{\odot}(R / \mathrm{pc})^{1.42}$. The red dashed line is the best fit to our sample, $M \propto R^{2.38 \pm 0.10}$.

The mass-radius diagram is also a useful tool to investigate clumps that are likely to form high-mass stars. The vast majority of these clumps can form high-mass objects, following the empirical mass-radius thresholds determined by Kauffmann \& Pillai (2010) and Baldeschi et al. (2017). All but two clumps (G333.449-00.183 and G338.917+00.382) are above the former, and all but three clumps (G333.449-00.183, G338.917+00.382 and G343.938+00.097) are above the latter, which is a more stringent threshold.

To conclude this section, we have shown that the Larson relations do not describe the dynamical properties of an ensemble of (massive) clumps. In the next section, we explore some implications of this evidence.

\section{DEPENDENCES OF THE VIRIAL PARAMETER}

A consequence of the breakdown of the Larson relations in massive clumps is the dependence of the virial parameter with the dust properties of these regions (mass and radius). There is an observed trend of decreasing virial parameter at increasing mass, which is interpreted as the most massive regions also being the more gravitationally bound regions. For example, the massive star-forming regions analysed by Urquhart et al. (2014) showed a power-law form $\alpha_{\text {vir }} \propto M^{\alpha}$ with $\alpha=-0.53 \pm 0.16$. Similarly, Kauffmann et al. (2013) found a slope varying in the range $-1 \leq \alpha \leq-0.4$ among various surveys of massive clumps and cores.

As noted by Kauffmann et al. (2013), the slope $\alpha$ also depends on both the first and third Larson relations:

$$
\frac{\mathrm{d} \log (\alpha)}{\mathrm{d} \log (M)}=\left[2 \frac{\mathrm{d} \log (\sigma)}{\mathrm{d} \log (R)}+1-\frac{\mathrm{d} \log (M)}{\mathrm{d} \log (R)}\right]\left[\frac{\mathrm{d} \log (M)}{\mathrm{d} \log (R)}\right]^{-1} \text {. }
$$

If the size and velocity dispersion in star-forming regions are not correlated, then $\mathrm{d} \log (\sigma) / \mathrm{d} \log (R)=0$. Equation (1) becomes

$$
\frac{\mathrm{d} \log (\alpha)}{\mathrm{d} \log (M)}=\left[1-\frac{\mathrm{d} \log (M)}{\mathrm{d} \log (R)}\right]\left[\frac{\mathrm{d} \log (M)}{\mathrm{d} \log (R)}\right]^{-1},
$$

which implies that the slope of the $\alpha_{\text {vir }}$-mass diagram depends only on the slope of the mass-radius diagram.

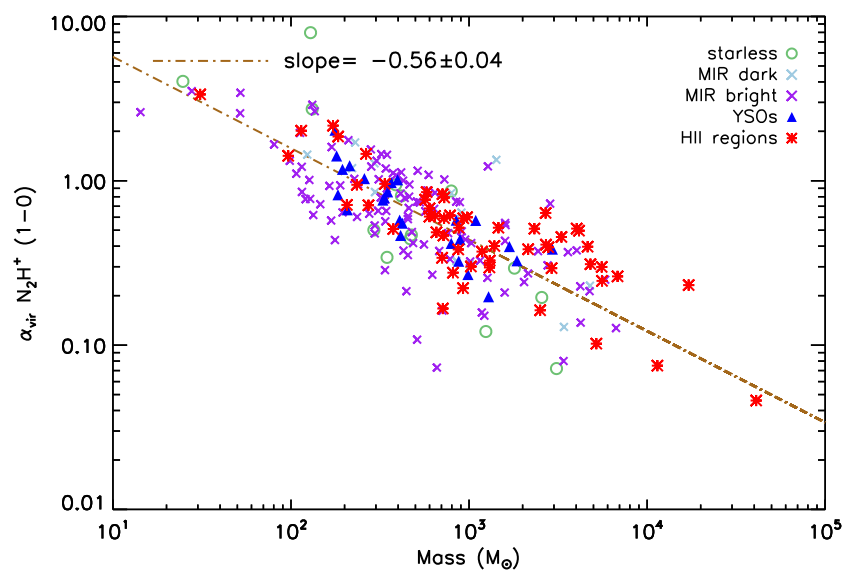

Figure 9. Virial parameter distribution as a function of mass. The red dashed line is the best fit to our data, which gives a slope $\alpha=-0.56 \pm 0.04$.

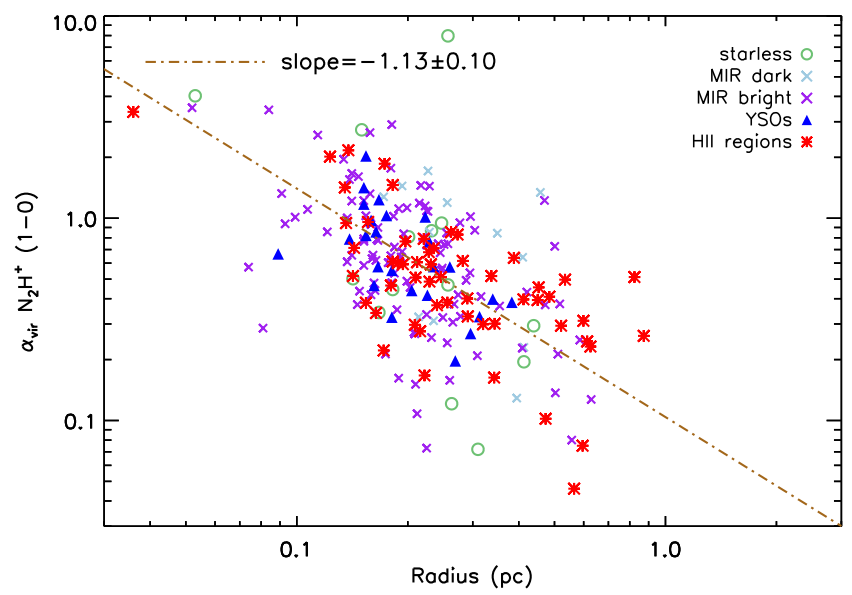

Figure 10. Distribution of the virial parameter as a function of clump radius $R$. The best-fitting line has a slope of $\alpha_{r}=-1.13 \pm 0.10$.

Applying equation (2) to our clumps, because the mass-radius slope is $\delta=2.38 \pm 0.10$ (see Section 4.3), the predicted $\alpha_{\text {vir }}$-mass slope is $\alpha-0.58 \pm 0.02$. In Fig. 9, we show the $\alpha_{\text {vir }}$ versus mass diagram. We found a slope $\alpha=-0.56 \pm 0.04$, in agreement with the prediction of equation (2). The fit is robust to the estimation of the uncertainties, with a variation of less than $\simeq 1$ per cent, assuming a variation of \pm 10 per cent on the errors associated with $M$ and $\alpha_{\text {vir }}$.

Similarly, the slope of the virial parameter-radius diagram is

$\frac{\mathrm{d} \log (\alpha)}{\mathrm{d} \log (R)}=2 \frac{\mathrm{d} \log (\sigma)}{\mathrm{d} \log (R)}+1-\frac{\mathrm{d} \log (M)}{\mathrm{d} \log (R)}$

When the first Larson relation is not valid, the slope depends, again, only on the slope of the mass-radius diagram.

The slope of the $\alpha_{\text {vir }}$-radius diagram predicted from equation (3) is $\alpha_{r}=-1.38 \pm 0.06$. The $\alpha_{\text {vir }}$-radius diagram is shown in Fig. 10 and the slope is $\alpha_{r}=-1.13 \pm 0.10$, which is slightly lower than the predicted value. This fit is also the most sensitive to the estimation of the uncertainties. A variation of \pm 10 per cent on the errors associated with $R$ and $\alpha_{\text {vir }}$ leads to a difference of more than 50 per cent in $\alpha_{r}$, which varies in the range $-0.97 \pm 0.10 \leq \alpha_{r} \leq$ $-1.50 \pm 0.12$, within the prediction of equation (3).

These results show that in our sample the kinematic properties of the clumps do not affect the distribution of the virial parameter, which are driven by the dust properties (mass and size) of these 
sources. In other words, the concept of virial equilibrium can be misleading: in a sample that violates the three Larson relations, the virial parameter varies independently of the kinetic energy of the sources.

Note that these results are valid for an ensemble of clumps, where the non-thermal motions are estimated with a single gas tracer. However, this common choice biases the observations towards regions with similar volume densities within each clump, regardless of the physical properties of the clump (i.e. mass and size). The correlation between $\alpha_{\text {vir }}$ and mass seems to disappear when different surveys of clumps and cores observed with different tracers are combined (Kauffmann et al. 2013). These results might differ from the analysis of the energy balance within single star-forming regions. They might all be near virial equilibrium, as predicted by, for example, Lee \& Hennebelle (2016), but the kinetic energy in the more massive clumps might not be properly measured.

The virial parameter determined for an ensemble of massive clumps might not be a good descriptor of clump dynamics, which is a hypothesis that we explore in the next section.

\section{INFALLING PROPERTIES}

If the virial parameter is not a good descriptor of the energy balance in an ensemble of massive clumps, then these regions might be gravitationally bound and collapsing independently of the value of $\alpha_{\text {vir }}$. One way to explore this hypothesis is to compare the properties of clumps that show signs of infall motions with clumps that have no such signatures.

The MALT90 survey also observed the $\mathrm{HCO}^{+}(1-0)$ transition, an optically thick line and a good tracer of infall motions (Fuller, Williams \& Sridharan 2005; Rygl et al. 2013; He et al. 2015; Traficante et al. 2017). These motions can be identified by looking at blue asymmetries in the spectra corresponding to single-peaked $\mathrm{N}_{2} \mathrm{H}^{+}(1-0)$ spectra, which avoids the risk that asymmetries in the $\mathrm{HCO}^{+}(1-0)$ spectra might be the result of obscuration by surrounding filaments (Chira et al. 2014).

We identified by eye all clumps with well-defined infall signatures - that is, all clumps with double-peaked blue-asymmetric $\mathrm{HCO}^{+}$ (1-0) spectra - and we found 21 clumps with such properties. This number likely represents a subsample of clumps that have parsecscale infall motions. Many clumps present red-asymmetric $\mathrm{HCO}^{+}$ (1-0) spectra despite the presence of infall motions that can be identified with blue-asymmetric spectra in higher-density tracers (Wyrowski et al. 2016). Also, infalling clumps might not always show the expected blue-asymmetric line profiles (Smith et al. 2013). At the same time, well-defined blue asymmetries in the $\mathrm{HCO}^{+}(1-0)$ spectra are clear signatures of infall motions and can be modelled to infer infall parameters (see the next section). Therefore, we restrict the analysis to this subsample of 21 clumps to analyse the main infall properties of our sample. These clumps are divided into ten protostar MIR bright, four YSOs and seven $\mathrm{H}$ II regions.

The clumps with infall signatures span two orders of magnitude in mass, and the virial parameter varies in the range $0.10 \leq \alpha_{\mathrm{vir}} \leq 1.96$, similar to the $\alpha_{\text {vir }}$ distribution of the rest of the sample, with four clumps that have $\alpha_{\text {vir }} \geq 1$. The differences between these clumps and the subsample of clumps with no blue-asymmetric $\mathrm{HCO}^{+}$spectra are not statistically significant. A Kolmogorov-Smirnov test, which gives the probability that two samples come from the same distribution, gives a 71 per cent probability that the distribution of the virial parameter of infalling clumps is similar to that of the other clumps. A Student $t$-test, which gives the probability that two samples have the same mean, gives a probability of 61 per cent that the means
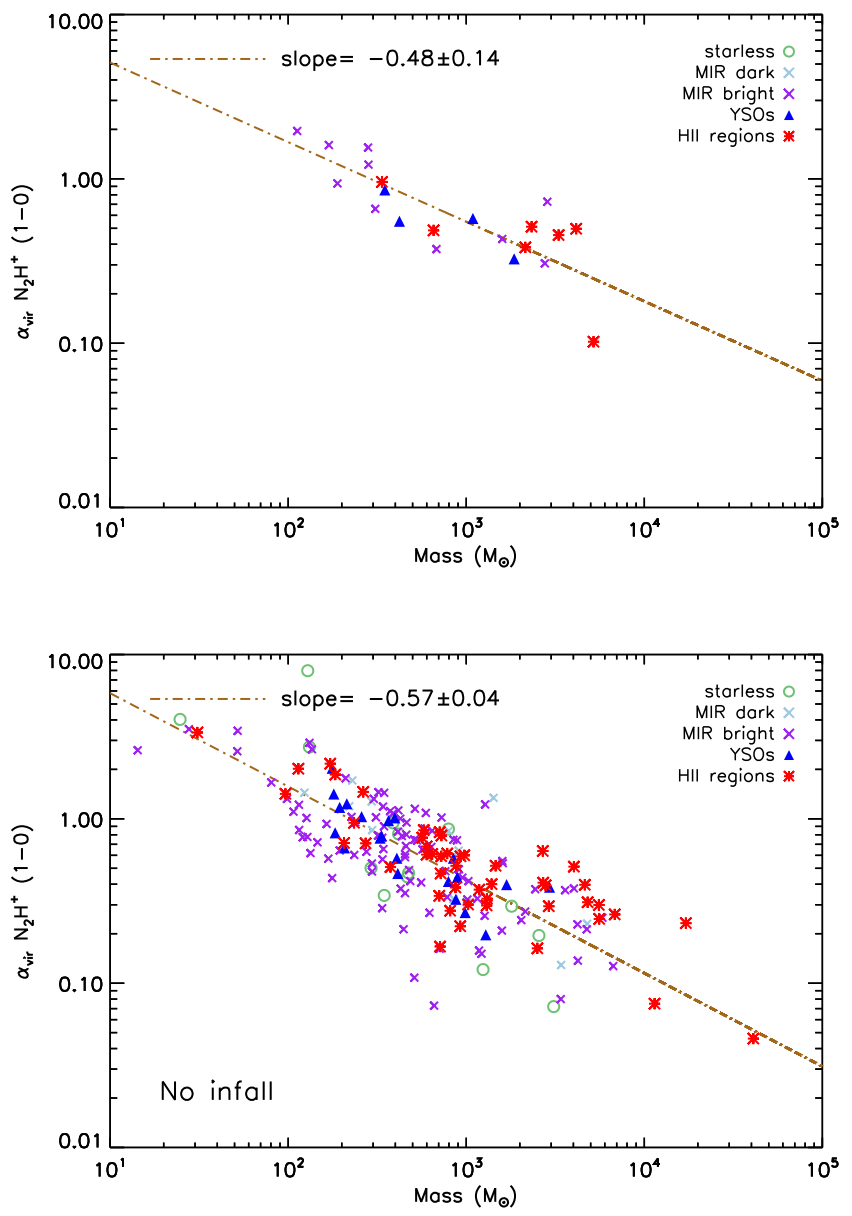

Figure 11. Upper panel: $\alpha_{\text {vir }}$ versus mass diagram for the 21 clumps that show evidence of infalling motions in their $\mathrm{HCO}^{+}(1-0)$ spectra. Lower panel: same diagram for the remaining 192 clumps of the sample. The slopes of the best linear fit in the two plots are the same as the uncertainties, suggesting that the clump dynamics is independent of the values of $\alpha_{\text {vir }}$.

of the virial parameter distributions in the two subsamples are the same.

Fig. 11 shows the $\alpha_{\text {vir }}$ versus mass diagrams for the 21 clumps with blue-asymmetric $\mathrm{HCO}^{+}$spectra (upper panel) and for the remaining 192 clumps (lower panel), with the respective fits. The linear fits in the diagrams in Fig. 11 have very similar slopes within the uncertainties, suggesting that the observed values of the virial parameter are independent of the dynamics of the clumps.

\subsection{Properties of infalling gas}

Clumps with double-peaked blue-asymmetric spectra can be modelled to obtain properties such as the infall velocity $v_{\text {in }}$ and mass accretion rate $\dot{M}$ using the two-layer model of Myers et al. (1996), for example. In this model, the line intensities of the red and blue peaks, as well as that of the corresponding dip of the central velocity of the clump (deduced from an optically thin line such as $\mathrm{N}_{2} \mathrm{H}^{+}$), are used to estimate the infall velocity:

$v_{\text {in }}=\frac{\sigma^{2}}{v_{\text {red }}-v_{\text {blue }}} \ln \left\{\frac{1+\exp \left[\left(T_{\text {blue }}-T_{\text {dip }}\right) / T_{\text {dip }}\right]}{1+\exp \left[\left(T_{\text {red }}-T_{\text {dip }}\right) / T_{\text {dip }}\right]}\right\}$.

Here, $v_{\text {red }}$ and $v_{\text {blue }}$ are the velocities of the blue and red peaks, respectively, $T_{\text {blue }}$ and $T_{\text {red }}$ are the main beam temperatures of the blue and red peaks, respectively, and $T_{\text {dip }}$ is the main beam temperature 
Table 4. Parameters of the 21 clumps derived from double-peaked, blueshifted $\mathrm{HCO}^{+}(1-0)$ spectra. In columns $2-4, v_{\text {in }}, \dot{M}$ and $\epsilon$ are the infall velocity, mass accretion rate and efficiency, respectively. The uncertainties have been propagated from the uncertainties on $M, R$ and $\sigma$ shown in Table 1, assuming a further uncertainty of 25 per cent on the estimation of $v_{\text {red }}$ and $v_{\text {blue }}$ because of the resolution of the smoothed spectra used to estimate the velocities (see Section 2.4). We obtained uncertainties of 50, 60 and 75 per cent on the estimation of $v_{\text {in }}, \dot{M}$ and $\epsilon$ respectively.

\begin{tabular}{lccc}
\hline Clump name & $\begin{array}{c}v_{\text {in }} \\
\left(\mathrm{km} \mathrm{s}^{-1}\right)\end{array}$ & $\begin{array}{c}\dot{M} \\
\left(10^{-3} \mathrm{M}_{\odot} \mathrm{yr}^{-1}\right)\end{array}$ & $\epsilon$ \\
\hline G309.422-00.622 & $1.01(0.51)$ & $7.22(4.33)$ & $0.11(0.08)$ \\
G316.085-00.674 & $0.26(0.13)$ & $0.68(0.41)$ & $8.80(6.60)$ \\
G316.140-00.504 & $0.85(0.43)$ & $28.65(17.19)$ & $0.15(0.11)$ \\
G320.285-00.309 & $0.53(0.27)$ & $11.90(7.14)$ & $3.08(2.31)$ \\
G321.935-00.007 & $0.26(0.13)$ & $1.63(1.98)$ & $11.39(8.54)$ \\
G322.520+00.637 & $0.22(0.11)$ & $1.27(0.76)$ & $10.81(8.11)$ \\
G327.393+00.199 & $0.19(0.10)$ & $2.45(1.47)$ & $41.37(31.03)$ \\
G327.403+00.444 & $0.66(0.33)$ & $17.11(10.27)$ & $1.50(1.13)$ \\
G331.132-00.245 & $0.21(0.11)$ & $6.11(3.67)$ & $87.48(65.61)$ \\
G331.708+00.583 & $0.55(0.28)$ & $9.65(5.79)$ & $3.80(2.85)$ \\
G331.723-00.203 & $0.74(0.37)$ & $4.23(2.54)$ & $0.65(0.49)$ \\
G332.604-00.168 & $0.21(0.11)$ & $0.73(0.44)$ & $21.47(16.10)$ \\
G338.927+00.632 & $1.43(0.72)$ & $45.83(27.50)$ & $0.15(0.11)$ \\
G339.476+00.185 & $1.51(0.76)$ & $36.06(21.64)$ & $0.17(0.13)$ \\
G339.924-00.084 & $0.51(0.26)$ & $9.21(5.53)$ & $1.60(1.20)$ \\
G341.215-00.236 & $0.20(0.10)$ & $1.73(1.04)$ & $16.45(12.34)$ \\
G342.822+00.382 & $0.33(0.17)$ & $3.87(2.32)$ & $4.26(3.20)$ \\
G343.520-00.519 & $0.33(0.17)$ & $2.13(1.28)$ & $5.39(4.04)$ \\
G343.756-00.163 & $0.23(0.12)$ & $3.30(1.98)$ & $14.20(10.65)$ \\
G344.101-00.661 & $1.13(0.57)$ & $7.00(4.20)$ & $0.30(0.23)$ \\
G344.221-00.594 & $0.24(0.12)$ & $1.61(0.97)$ & $15.72(11.79)$ \\
\hline
\end{tabular}

Table 5. Mean infall parameters of the 21 clumps with infall signatures divided by different evolutionary phases. The columns show the clump evolutionary phase, the number of clumps in each phase, the mean infall velocity and the mean mass accretion rate, respectively.

\begin{tabular}{lccc}
\hline Clump phase & Count & $\begin{array}{c}\bar{v}_{\text {in }} \\
\left(\mathrm{km} \mathrm{s}^{-1}\right)\end{array}$ & $\begin{array}{c}\bar{M} \\
\left(10^{-3} \mathrm{M}_{\odot} \mathrm{yr}^{-1}\right)\end{array}$ \\
\hline 24- $\mu$ m bright & 10 & 0.54 & 7.82 \\
YSOs & 4 & 0.51 & 5.25 \\
H II regions & 7 & 0.60 & 14.74 \\
\hline
\end{tabular}

of the valley between the two peaks. To obtain these parameters, the spectra of the 21 clumps have been fitted with a double-Gaussian model using the mpfitfun IDL routine (Markwardt 2009). The spectra with the double-Gaussian fits are given in Appendix C.

The infall velocity varies in the range $0.03 \leq v_{\text {in }} \leq 2.75 \mathrm{~km} \mathrm{~s}^{-1}$, with an average value of $v_{\text {in }}=0.55 \mathrm{~km} \mathrm{~s}^{-1}$, in line with similar estimates in massive star-forming regions (Fuller et al. 2005; Rygl et al. 2013; Traficante et al. 2017). The value for each clump is in Table 4.

The mass accretion rate $\dot{M}$ can be evaluated by assuming spherical geometry as $\dot{M}=4 \pi R^{2} n_{\mathrm{H}_{2}} \mu m_{\mathrm{H}} v_{\text {in }}$ (Myers et al. 1996), where $m_{\mathrm{H}}$ is the hydrogen mass, $\mu=2.33$ is the molecular weight and $n_{\mathrm{H}_{2}}$ is the volume density. It is in the range $0.68 \leq \dot{M} \leq 45.8 \times 10^{-3}$ $\mathrm{M}_{\odot} \mathrm{yr}^{-1}$ (Table 4), with an average value of $\dot{M}=9.6 \times 10^{-3}$ $\mathrm{M}_{\odot} \mathrm{yr}^{-1}$, comparable with similar results for massive protostellar clumps (e.g. Peretto et al. 2013; Rygl et al. 2013).

In Table 5, we show the average values of $v_{\text {in }}$ and $\dot{M}$ for the various evolutionary phases. There is an indication that $\dot{M}$ is higher in $\mathrm{H}_{\mathrm{II}}$ regions than in the rest of the sample, suggesting an increasing of

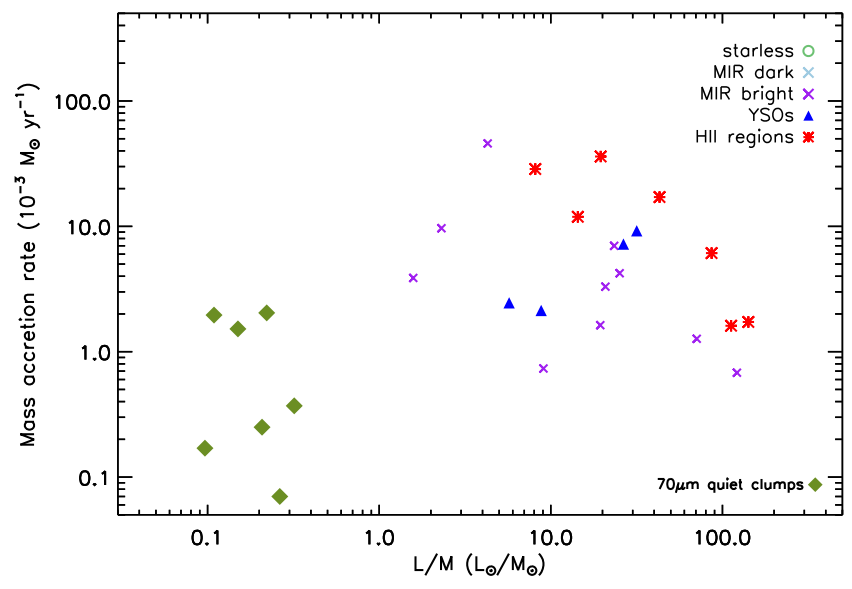

Figure 12. Mass accretion rate as a function of the $L / M$ ratio, an indicator of clump evolution. Our sample of 21 clumps has been combined with the survey of 70- $\mu \mathrm{m}$ quiet clumps in Traficante et al. (2017, green diamonds). These data show a moderate correlation $(\rho=0.44)$ and suggest that the mass accretion rate might increase with evolution.

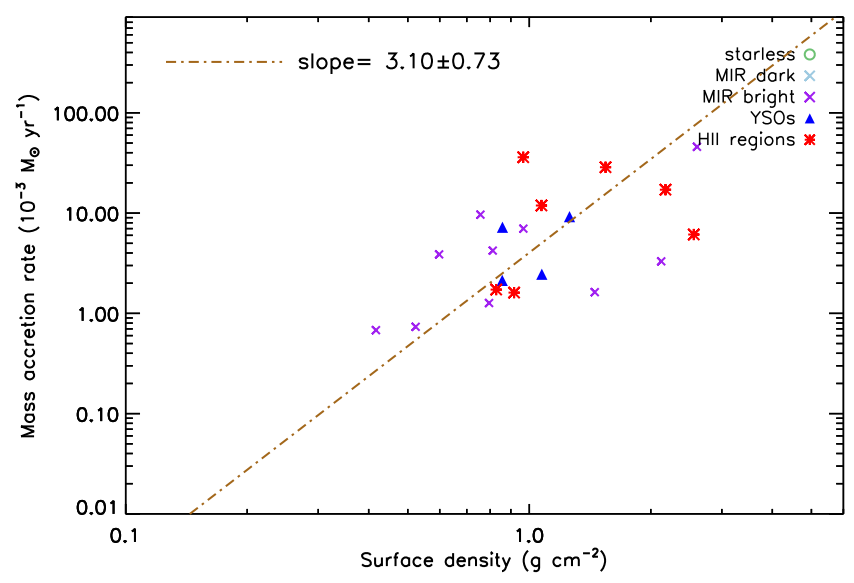

Figure 13. Mass accretion rate as a function of surface density. The Pearson correlation coefficient is 0.61 , suggesting a good correlation between these two quantities. The best fit gives a slope of $3.10 \pm 0.73$, suggesting an increase of $\dot{M}$ as a function of $\Sigma$.

the accretion rate with evolution. Because we do not observe clumps at the earliest evolutionary stages with a clear hint of infall motions in our data, in order to investigate this trend we have combined our data with the sample of seven $70-\mu \mathrm{m}$ quiet massive clumps studied by Traficante et al. (2017), who measured their mass accretion rates. In Fig. 12, we plot $\dot{M}$ against the quantity $L / M$. There is a large scatter among the sources, but the correlation is not irrelevant $(\rho=0.44)$. With an average accretion rate for the $70-\mu \mathrm{m}$ quiet clumps of $0.91 \times 10^{-3} \mathrm{M}_{\odot} \mathrm{yr}^{-1}$, this diagram indicates that $\dot{M}$ increases with evolution in these massive objects.

The mass accretion rate is proportional to the surface density $(\rho=0.61)$, as shown in Fig. 13. This result implies that higherdensity regions sustain a higher accretion rate, a point that we discuss further in Section 7.2.

\section{ORIGIN OF NON-THERMAL MOTIONS}

In comparison with the non-thermal motions found in GMCs, our clumps have an excess of kinetic energy at small radii (Fig. 4), in agreement with the findings of Ballesteros-Paredes et al. (2011, and 
references therein). Larson (1981) himself noted that the relation breaks down at the size of the clumps/cores and that the inner parts of massive star-forming regions tend to have higher velocity dispersion at a given radius. The kinetic energy excess in this ensemble of clumps should have a different origin from shock turbulence. In this section, we investigate the possible origins of the observed non-thermal motions in these objects.

A possible explanation for the origin of non-thermal motions in massive clumps is given by the model of Murray \& Chang (2015), who break down the assumption that collapsing regions are in hydrostatic equilibrium. Instead, the turbulent velocity is adiabatically heated by the collapse itself, following the evolution of the system. Combined with the back-pressure generated by turbulence, Murray \& Chang (2015) predicted a power-law form for the first Larson relation of $R \propto \sigma^{0.2-0.3}$. This model successfully predicts a deviation of the first Larson relation in massive star-forming regions as found by, for example, Caselli \& Myers (1995) and Shirley et al. (2003). However, in agreement with the findings of BallesterosParedes et al. (2011) and Traficante et al. (2018), our observations suggest that there is no correlation between the velocity dispersion and the size of an ensemble of clumps.

Non-thermal motions in massive star-forming objects can also be driven by stellar feedbacks such as protostellar jets/outflows (e.g. Federrath 2016). However, Figs 4 and 5 show that the velocity dispersion in starless clumps, which are less affected by stellar feedbacks, is similar to that observed in more evolved clumps, and the quantity $\sigma / R^{0.5}$ is not constant. Even if stellar feedbacks play an important role in the observed non-thermal motions of protostars, these alone cannot explain the observed linewidth-size relation in all these clumps.

\subsection{Accretion-driven turbulence}

An alternative explanation to the observed supersonic motions is that these non-thermal velocities are the result of accretion-driven turbulence (Klessen \& Hennebelle 2010). This model predicts that (at least part of) the energy injected by the accretion into the system is converted into turbulent motions, which set up a Kolmogorov-like turbulent cascade. The large-scale fed accretion generates enough turbulence to produce supersonic motions in the high-density clumps.

If the energy injected by the infall motions is much lower than the turbulent dissipation rate, then the conversion of these motions into turbulent energy cannot maintain the turbulent cascade, which will rapidly dissipate. Therefore, the observed non-thermal motions would not be the result of a turbulent cascade, and they would not follow a Larson-like relation.

The key parameters to evaluate the energy injected by the accretion and the turbulent dissipation rate are the scale at which the turbulence is driven and the mass of the infalling gas. We consider that the clumps are globally collapsing as a whole (as observed, for example, in Traficante et al. 2017), and the representative scale is the scale of the clumps.

With these assumptions, the turbulent dissipation rate is

$\dot{E}_{\text {dis }}=\frac{1}{2} \frac{M \sigma^{2}}{\tau_{\mathrm{d}}}=\frac{1}{2 \sqrt{3} L_{\mathrm{d}}} M \sigma^{3}$,

where $M$ is the total mass of the clump (Hennebelle \& Falgarone 2012). The turbulence decays in a turbulent crossing time $\tau_{\mathrm{d}}=L_{\mathrm{d}} / \sigma_{3 \mathrm{D}}$, where $\sigma_{3 \mathrm{D}}=\sqrt{3} \sigma$ is the three-dimensional velocity dispersion and $L_{\mathrm{d}}$ is the turbulence driving scale (see Hennebelle $\&$ Falgarone 2012, and references therein), the size of the clump.

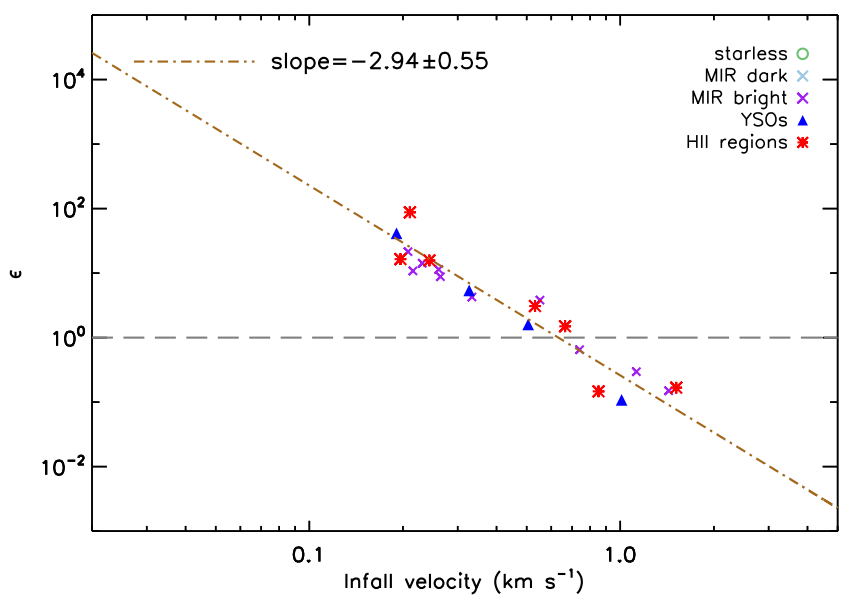

Figure 14. Efficiency $\epsilon$ as a function of the infall velocity. The red dashed line is the best fit to the data and shows that the efficiency decreases rapidly with the increase of the infall velocity. The black dashed line corresponds to $\epsilon=1$. The non-thermal motions observed in clumps with efficiency below this value might be the result of accretion-driven turbulence.

The energy injected by the accretion is $\dot{E}_{\text {inj }}=(1 / 2) \dot{M} v_{\text {in }}^{2}$ (Klessen \& Hennebelle 2010), where $\dot{M}$ has been evaluated from the mean density of the clumps as in Section 6.1. Defining the efficiency $\epsilon=\dot{E}_{\text {dis }} / \dot{E}_{\text {inj }}$, the conditions for accretion-driven turbulence are satisfied if $\epsilon \leq 1$ (Klessen \& Hennebelle 2010).

We evaluate $\dot{E}_{\mathrm{dis}}, \dot{E}_{\mathrm{inj}}$ and $\epsilon$ for the 21 clumps with defined infall velocities (Section 6.1). The efficiency as a function of the infall velocity is shown in Fig. 14. The efficiency decreases rapidly as the infall velocity increases, and it becomes less than 1 for the six clumps with the highest accretion rates and with infall velocities $v_{\text {in }} \geq 0.75 \mathrm{~km} \mathrm{~s}^{-1}$ (Table 4). For the majority of the clumps, the turbulent dissipation rate seems to be sufficiently high to dissipate the energy injected by the accretion.

The observed non-thermal motions can partly originate from turbulence driven by the accretion in clumps with high infall velocity and accretion rates. However, under the hypothesis that the driving scales are the clump scales that are globally collapsing as a whole, this mechanism alone cannot explain the supersonic non-thermal motions observed in clumps with infall velocity $v_{\text {in }}<0.75 \mathrm{~km} \mathrm{~s}^{-1}$.

\subsection{Gravity-driven non-thermal motions}

Non-thermal motions in star-forming regions might originate from gravity itself, which seems to play a dominant role in the evolution of molecular clouds able to form high-mass stars, down to $\simeq 0.1 \mathrm{pc}$ scales (Li \& Burkert 2016, 2017). In particular, non-thermal motions might originate from a hierarchical, global collapse of clouds and clumps (Ballesteros-Paredes et al. 2011). In this scenario, the supersonic motions are not hydrodynamical turbulence, but organized motions driven by gravity in multiple centres of collapse. This hypothesis implies that massive regions should develop a larger velocity dispersion for larger column densities (Ballesteros-Paredes et al. 2011), which might also explain the higher accretion rates observed in clumps with higher surface density (Fig. 13).

Fig. 15 shows the $\sigma$ versus $\Sigma$ diagram. The correlation is not strong, but there is a weak positive correlation $(\rho=0.30)$ and regions with higher surface density have, on average, higher velocity dispersion, suggesting that non-thermal motions might partly result from the large gravitational force acting in the system. 


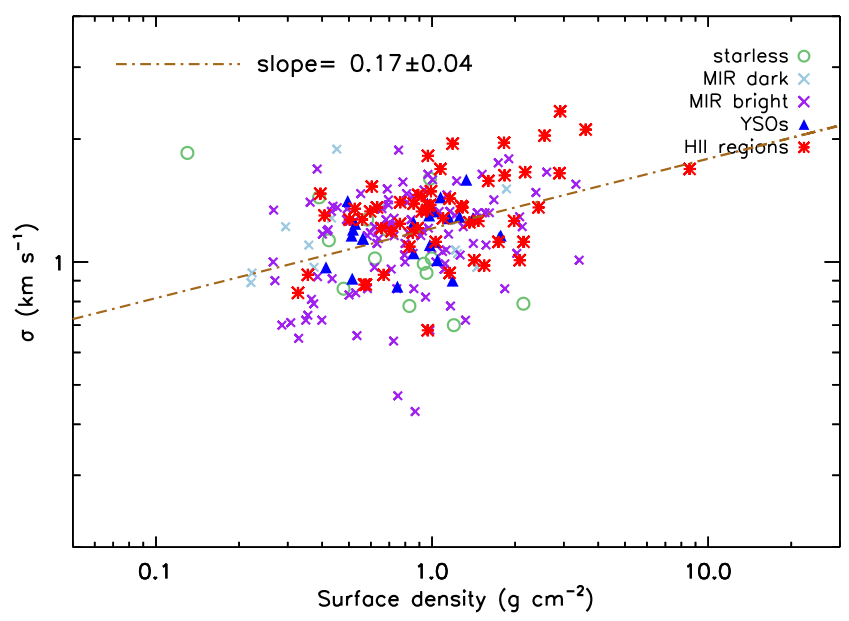

Figure 15. Velocity dispersion $\sigma$ as a function of surface density $\Sigma$. The positive correlation is weak, with a Pearson correlation coefficient of $\rho=0.30$, and the best fit gives a slope of $0.17 \pm 0.04$.

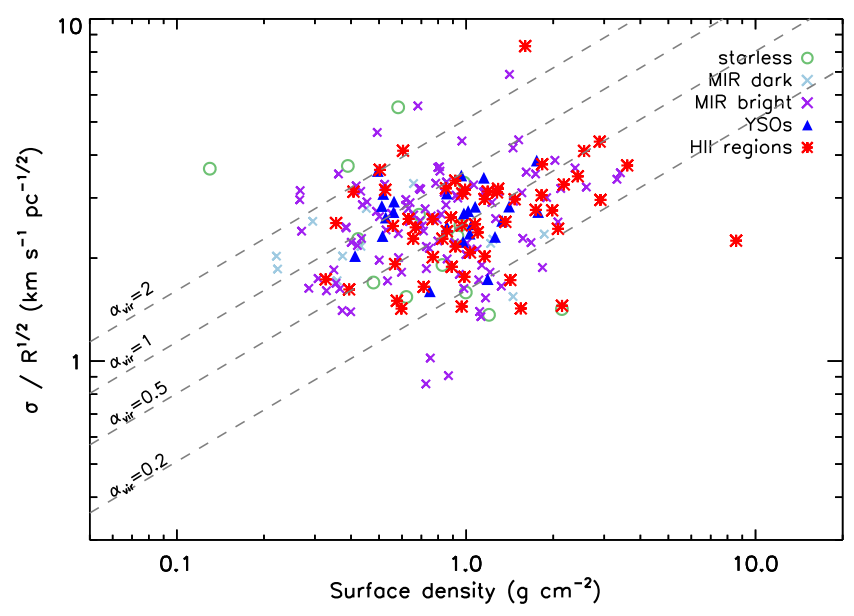

Figure 16. The Heyer plot showing the quantity $\sigma / R^{1 / 2}$ versus the surface density $\Sigma$. The dashed lines correspond to constant values of the virial parameter, from $\alpha_{\mathrm{vir}}=2$ (highest line) to $\alpha_{\mathrm{vir}}=0.2$ (lowest line). The correlation is weak, with a Pearson coefficient of $\rho=0.18$.

This model also considers that the system develops a Heyer-like relation $\sigma / R^{1 / 2} \propto \Sigma^{1 / 2}$ (Heyer et al. 2009). This relation is equivalent to a generalized first Larson relation for regions with different surface densities (Ballesteros-Paredes et al. 2011; Camacho et al. 2016). In this global collapse model, it is not the virial equilibrium that counts but the conservation of the total energy of the system. The virial parameter represents energy equipartition, which numerically is equivalent to $\alpha_{\mathrm{eq}}=2$ (Ballesteros-Paredes et al. 2011).

The Heyer plot for our clumps is shown in Fig. 16. The correlation between $\Sigma$ and the quantity $\sigma / R^{1 / 2}$ is relatively weak $(\rho=0.18)$. The majority of the clumps lie below the equipartition value and there is a significant spread across the diagram, which reflects the different values of the virial parameter (Section 4.2).

Fig. 17 shows the same Heyer plot as in Fig. 16, but limited to clumps with mass $M \geq 1000 \mathrm{M}_{\odot}$. The correlation in this case is higher $(\rho=0.40)$. We interpret this result as an indication that gravity drives, at least partially, the observed non-thermal motions,

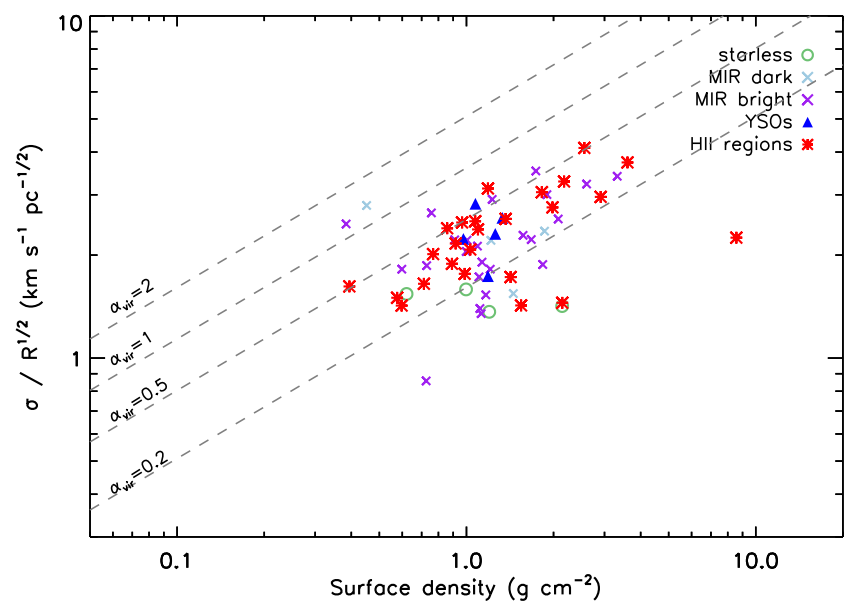

Figure 17. Same as in Fig. 16 but for clumps with $M \geq 1000 \mathrm{M}_{\odot}$. The correlation is higher, with a Pearson coefficient of $\rho=0.40$.

in particular in the more massive clumps of the sample. If gravity contributes to the generation of the observed kinetic energy, then this contribution is more dominant at higher masses, although all these massive clumps lie below the equipartition value. A possible explanation for the observed departure from the equipartition, at least for the subvirial clumps at the earliest phases of evolution, is that a collapsing region with a sufficiently low level of local turbulence can start in a subvirial state, and it can reach equipartition during its evolution (Ballesteros-Paredes et al. 2017). As discussed in Section 5, there might also be a fraction of kinetic energy not properly traced in these massive regions, which might explain the departure from the energy equipartition expected from the models. In the following subsection, we explore whether the magnetic pressure in these clumps is another valid explanation for this observed departure.

\subsection{Role of magnetic fields}

In this subsection, we estimate the possible contribution of the magnetic fields to the stability of these clumps.

In accordance with the above findings, these clumps, in particular the more massive clumps, might be undergoing gravitational collapse. However, these clumps might be sustained against collapse by strong magnetic pressure.

Crutcher (2012) has shown that, observationally, the magnetic field strength might not be sufficient to balance gravity in highdensity regions $\left(n \geq 300 \mathrm{~cm}^{-3}\right)$, although they might give a significant contribution in lower-density regions. There is an expected upper limit to the intensity of the magnetic field $B_{\mathrm{Cr}}$, which increases at increasing density as $B_{\mathrm{Cr}} \simeq \Sigma^{0.65}$ (Crutcher 2012). At the same time, Kauffmann et al. (2013) showed that the magnetic field strength required to maintain a clump in a hydrostatic equilibrium (equivalent to $\alpha_{\text {vir }}=2$ ) is proportional to the observed non-thermal motions following the relation:

$B_{M_{\mathrm{BE}}}=81 \mu \mathrm{G} \frac{M_{\phi}}{M_{\mathrm{BE}}}\left(\frac{\sigma}{\mathrm{km} \mathrm{s}^{-1}}\right)^{2}\left(\frac{R}{\mathrm{pc}}\right)^{-1}$.

Here, $M_{\phi} / M_{\mathrm{BE}}$ is the ratio between the magnetic flux mass and the mass of a sphere in hydrostatic equilibrium (a Bonnor-Ebert sphere), and it is proportional to $2 / \alpha_{\text {vir }}-1$ (Kauffmann et al. 2013). 


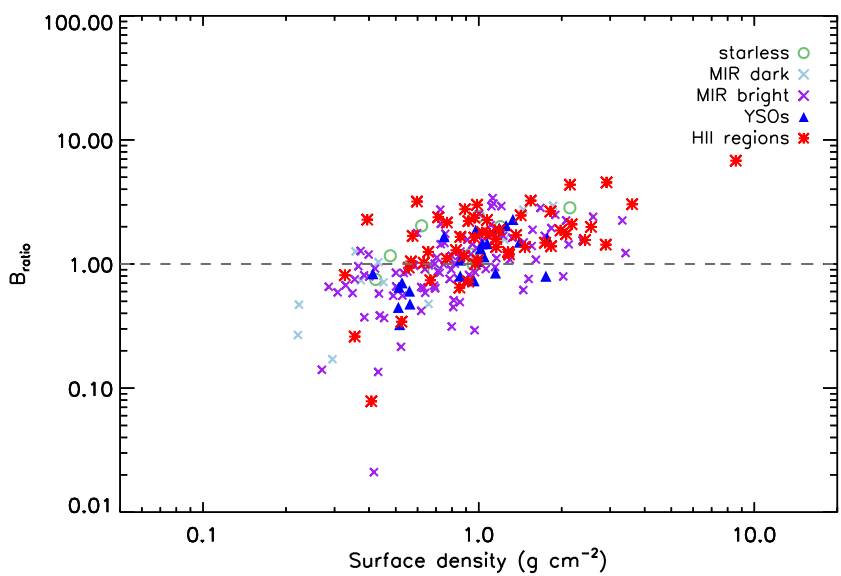

Figure 18. The $B_{\text {ratio }}=B_{M_{\mathrm{BE}}} / B_{\mathrm{Cr}}$ ratio as a function of surface density. To be stabilized by magnetic fields, clumps with $B_{\text {ratio }}>1$ (black dotted line) require magnetic fields stronger than the maximum values estimated by Crutcher (2012).

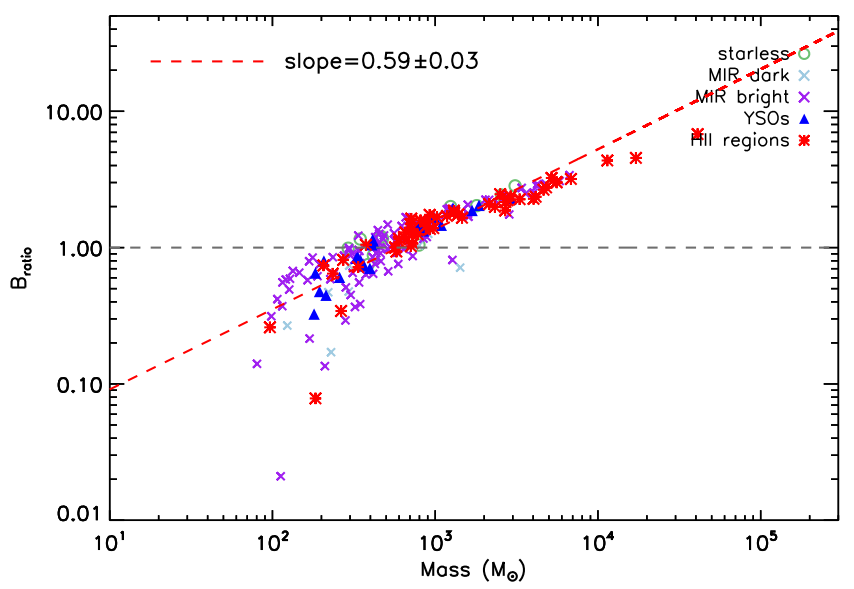

Figure 19. $B_{\text {ratio }}$ as a function of the clump mass. The correlation is strong (Pearson coefficient $\rho=0.80$ ) and shows that the magnetic fields required to stabilize the clumps increase significantly with the mass of the clumps. For $M \geq 1000 \mathrm{M}_{\odot}$, exceptionally high magnetic fields are required to slow down the collapse.

Fig. 18 shows the quantity $B_{\text {ratio }}=B_{M_{\mathrm{BE}}} / B_{\mathrm{Cr}}$ as a function of the surface density for the 199 clumps with $\alpha_{\text {vir }} \leq 2$. Almost 40 per cent of these clumps (i.e. 80 ) lie below the threshold $B_{\text {ratio }}=1$. The majority of them have $B_{\text {ratio }}>1$, and the implication is that only exceptionally high magnetic fields can stabilize their collapse.

Fig. 19 shows $B_{\text {ratio }}$ as a function of the mass of the clumps. The correlation is strong $(\rho=0.80)$. This diagram shows that the intensity of the magnetic fields required to stabilize a collapsing clump exceeds the threshold estimated by Crutcher (2012) if the clump has a mass $M \geq 1000 \mathrm{M}_{\odot}$.

The results suggest that magnetic fields might be relevant in some of these clumps but for the majority of them, in particular the more massive clumps, this mechanism alone cannot sustain the collapse at clump scales. This analysis does not exclude the fact that at the scales of the inner cores the magnetic fields might play a relevant role and act against gravitational collapse (e.g. Fontani et al. 2016). Further observations at high resolution, with instruments such as
ALMA or NOEMA, are required to investigate the properties of these clumps at the core scales.

\section{CONCLUSIONS}

We have discussed the validity of the three Larson relations and the implications of the results for a large sample of 213 massive clumps at different evolutionary stages. These clumps have been obtained by combining the Hi-GAL catalogue (Elia et al. 2017) with the MALT90 survey of 3-mm emission lines (Jackson et al. 2013). They have been selected to be a sample of sources with well-known distances, dust emission properties and $\mathrm{N}_{2} \mathrm{H}^{+}$(1-0) emission, the latter used to extract the gas kinematics. The sample has been divided into five evolutionary stages, and we have obtained 14 starless, 12 protostar MIR dark, 106 protostar MIR bright, 25 YSOs and $56 \mathrm{H}$ II regions. They are all located in the IV Quadrant and the vast majority of these clumps will likely form high-mass stars, based on the Kauffmann et al. (2013) and Baldeschi et al. (2017) selection criteria for massive star formation.

We have shown that the three Larson relations do not describe the properties of an ensemble of massive clumps, independently of the evolutionary stage of these objects. At these scales, $\sigma$ is not proportional to the radius $R$ (the first Larson relation), these clumps are not in virial equilibrium (the second Larson relation) and these clumps have no constant surface density (the third Larson relation).

We have demonstrated that the absence of a scaling relation between $\sigma$ and $R$ implies that the virial parameter $\alpha_{\text {vir }}$, defined as the ratio between kinetic energy $E_{\text {kin }}$ and gravitational energy $E_{G}$, decreases with mass and radius only as a function of the gravitational content of the clumps, independently of their kinetic energy.

A consequence of these findings is that the measured virial parameter is not a good descriptor of the clump dynamics. In fact, the virial values in clumps that show evidence of infalling motions (measured from blue-asymmetric $\mathrm{HCO}^{+}(1-0)$ spectra) are statistically indistinguishable from the values of the rest of the sample. This also suggests that all these clumps might be dynamically active, even those without clear evidence in their $\mathrm{HCO}^{+}(1-0)$ spectra.

We have shown that the observed non-thermal motions in massive clumps are not likely to be the result of turbulent cascade, collapse in adiabatically heated regions or accretion-driven turbulence. The velocity dispersion and mass accretion rate moderately correlate with surface density, which suggests that the gravitational collapse contributes at least partially to the variation of the observed non-thermal motions, in agreement with global collapse models (Ballesteros-Paredes et al. 2011). The gravitational collapse seems to play a dominant role, particularly in the more massive clumps of our sample $\left(M \geq 1000 \mathrm{M}_{\odot}\right)$, although these clumps all are subvirial and not in energy equipartition, as predicted in many gravitationally driven collapse models.

We have also shown that, on average, magnetic fields do not contribute significantly to the stability of these clumps, and exceptionally strong magnetic fields would be required to stabilize the clumps with $M \geq 1000 \mathrm{M}_{\odot}$.

\section{ACKNOWLEDGEMENTS}

AT would like to thank J. Kauffmann for useful and stimulating discussions that led to some of the results in this work. This work has benefitted from research funding from the European Community's 
Seventh Framework Programme. ADC acknowledges the support of the UK Science and Technology Facilities Council consolidated grant ST/N000706/1.

\section{REFERENCES}

Baldeschi A. et al., 2017, MNRAS, 466, 3682

Ballesteros-Paredes J., 2006, MNRAS, 372, 443

Ballesteros-Paredes J., Hartmann L. W., Vázquez-Semadeni E., Heitsch F., Zamora-Avilés M. A., 2011, MNRAS, 411, 65

Ballesteros-Paredes J., D’Alessio P., Hartmann L., 2012, MNRAS, 427, 2562

Ballesteros-Paredes J., Vazquez-Semadeni E., Palau A., Klessen R. S., 2017, preprint (arXiv:1710.07384)

Beckwith S. V. W., Sargent A. I., Chini R. S., Guesten R., 1990, AJ, 99, 924

Bertoldi F., McKee C. F., 1992, ApJ, 395, 140

Bonnell I. A., Vine S. G., Bate M. R., 2004, MNRAS, 349, 735

Brand J., Blitz L., 1993, A\&A, 275, 67

Camacho V., Vazquez-Semadeni E., Ballesteros-Paredes J., Gómez G. C., Fall S. M., Mata-Chávez M. D., 2016, ApJ, 833, 113

Caselli P., Myers P. C., 1995, ApJ, 446, 665

Chira R. A., Smith R. J., Klessen R. S., Stutz A. M., Shetty R., 2014, MNRAS, 444, 874

Crutcher R. M., 2012, ARA\&A, 50, 29

Csengeri T. et al., 2014, A\&A, 565, A75

Egan M. P., Price S. D., Kraemer K. E., 2003, Bull. Amer. Astron. Soc., 35, 1301

Elia D. et al., 2017, MNRAS, 471, 100

Ellsworth-Bowers T. P. et al., 2015, ApJ, 805, 157

Federrath C., 2016, J. Phys.: Conf. Ser., 719, 012002

Fontani F. et al., 2016, A\&A, 593, L14

Fuller G. A., Williams S. J., Sridharan T. K., 2005, A\&A, 442, 949

Griffin M. et al., 2008, Proc. SPIE, 7010, 701006

Gutermuth R. A., Heyer M., 2015, ApJ, 149, 64

He Y-X. et al., 2015, MNRAS, 450, 1926

Hennebelle P., Falgarone E., 2012, A\&AR, 20, 55

Heyer M. H., Brunt C. M., 2004, ApJ, 615, L45

Heyer M. H., Krawczyk C., Duval J., Jackson J. M., 2009, ApJ, 699, 1092

Hoare M. G., Kurtz S. E., Lizano S., Keto E., Hofner P., 2007, in Reipurth B., Jewitt D., Kei K., eds, Protostars and Planets V. University of Arizona Press, Tucson, p. 181

Hou L. G., Han J. L., Shi W. B., 2009, A\&A, 499, 473

Jackson J. M. et al., 2006, ApJS, 163, 145

Jackson J. et al., 2013, PASA, 30, e057

Juvela M. et al., 2015, A\&A, 584, A94

Kauffmann J., Pillai T., 2010, ApJ, 723, L7

Kauffmann J., Pillai T., Shetty R., Myers P. C., Goodman A. A., 2010, ApJ, 716,433

Kauffmann J., Pillai T., Goldsmith P. F., 2013, ApJ, 779, 185

Kegel W. H., 1989, A\&A, 225, 517

Klessen R. S., Hennebelle P., 2010, A\&A, 520, A17

König C. et al., 2017, A\&A, 599, A139

Kritsuk A. G., Lee C. T., Norman M. L., 2013, MNRAS, 436, 3247

Larson R. B., 1981, MNRAS, 194, 809

Lee Y.-N., Hennebelle P., 2016, A\&A, 591, A30

Li G.-X., Burkert A., 2016, MNRAS, 461, 3027

Li G.-X., Burkert A., 2017, MNRAS, 464, 4096

Lombardi M., Alves J., Lada C. J., 2010, A\&A, 519, L7

Lumsden S. L., Hoare M. G., Urquhart J. S., Oudmaijer R. D., Davies B., Mottram J. C., Cooper H. D. B., Moore T. J. T., 2013, ApJS, 208, 11

Mac Low M.-M., Klessen R. S., 2004, Rev. Mod. Phys., 76, 125

Markwardt C. B., 2009, in Bohlender D. A., Durand D., Dowler P., eds., ASP Conf. Ser. Vol. 411, Astronomical Data Analysis Software and Systems XVIII. Astron. Soc. Pac., San Francisco, p. 251
McKee C. F., Ostriker E. C., 2007, ARA\&A, 45, 565

McKee C. F., Tan J. C., 2003, ApJ, 585, 850

Miettinen O., 2014, A\&A, 562, A3

Molinari S., Pezzuto S., Cesaroni R., Brand J., Faustini F., Testi L., 2008, A\&A, 481, 345

Molinari S. et al., 2010, PASP, 122, 314

Molinari S., Schisano E., Faustini F., Pestalozzi M., di Giorgio A. M., Liu S., 2011, A\&A, 530, A133

Molinari S., Merello M., Elia D., Cesaroni R., Testi L., Robitaille T., 2016a, ApJ, 826, L8

Molinari S. et al., 2016b, A\&A, 591, A149

Murray N., Chang P., 2015, ApJ, 804, 44

Myers P. C., Mardones D., Tafalla M., Williams J. P., Wilner D. J., 1996, ApJ, 465, L133

Ossenkopf V., Henning T., 1994, A\&A, 291, 943

Padoan P., Nordlund A., 2002, ApJ, 576, 870

Paradis D. et al., 2010, A\&A, 520, L8

Peretto N. A. et al., 2013, A\&A, 555, A112

Plume R., Jaffe D. T., Evans N. J., II, Martín-Pintado J., Gómez-González J., 1997, ApJ, 476, 730

Poglitsch A. et al., 2010, A\&A, 518, L2

Ragan S. E., Bergin E. A., Gutermuth R. A., 2009, ApJ, 698, 324

Reid M. J. et al., 2009, ApJ, 700, 137

Russeil D. et al., 2011, A\&A, 526, A151

Rygl K. L. J., Wyrowski F., Schuller F., Menten K. M., 2013, A\&A, 549, A5

Schisano E. et al., 2014, ApJ, 791, 27

Schuller F. et al., 2009, A\&A, 504, 415

Shirley Y. L., Evans N. J., II, Young K. E., Knez C., Jaffe D. T., 2003, ApJS, 149,375

Smith R. J., Shetty R., Beuther H., Klessen R. S., Bonnell I. A., 2013, ApJ, 771,24

Solomon P. M., Rivolo A. R., Barrett J., Yahil A., 1987, ApJ, 319, 730

Svoboda B. E. et al., 2016, ApJ, 822, 59

Tan J. C., Beltrán M. T., Caselli P., Fontani F., Fuente A., Krumholz M. R., McKee C. F., Stolte A., 2014, in Beuther H., Klessen R. S., Dullemond C. P., Henning T., eds, Protostars and Planets VI. University of Arizona Press, Tucson, p. 149

Traficante A., Fuller G. A., Peretto N., Pineda J. E., Molinari S., 2015a, MNRAS, 451, 3089

Traficante A., Fuller G. A., Pineda J. E., Pezzuto S., 2015b, A\&A, 574, A119

Traficante A., Fuller G. A., Billot N., Duarte-Cabral A., Merello M., Molinari S., Peretto N., Schisano E., 2017, MNRAS, 470, 3882

Traficante A., Fuller G., Smith R. J., Billot N., Duarte-Cabral A., Peretto N., Molinari S., Pineda J. E., 2018, MNRAS, 473, 4975

Urquhart J. S. et al., 2014, MNRAS, 443, 1555

Urquhart J. S. et al., 2018, MNRAS, 473, 1059

Whitaker J. S., Jackson J. M., Rathborne J. M., Foster J. B., Contreras Y., Sanhueza P., Stephens I. W., Longmore S. N., 2017, AJ, 154, 140

Wood D. O. S., Churchwell E., 1989, ApJS, 69, 831

Wright E. L. et al., 2010, AJ, 140, 1868

Wu J., Evans N. J., II, Shirley Y. L., Knez C., 2010, ApJS, 188, 313

Wyrowski F. et al., 2016, A\&A, 585, A149

Zinnecker H., Yorke H. W., 2007, ARA\&A, 45, 481

\section{APPENDIX A: ALTERNATIVE SOURCE PHOTOMETRY}

In order to estimate the uncertainties associated with the chosen source extraction and photometry strategy described in Elia et al. (2017) and obtained using the Cutex algorithm (Molinari et al. 2011), we compared the results with the photometry obtained using an alternative method.

The alternative photometry has been carried out using Hyper, which performs elliptical aperture photometry in the presence of 

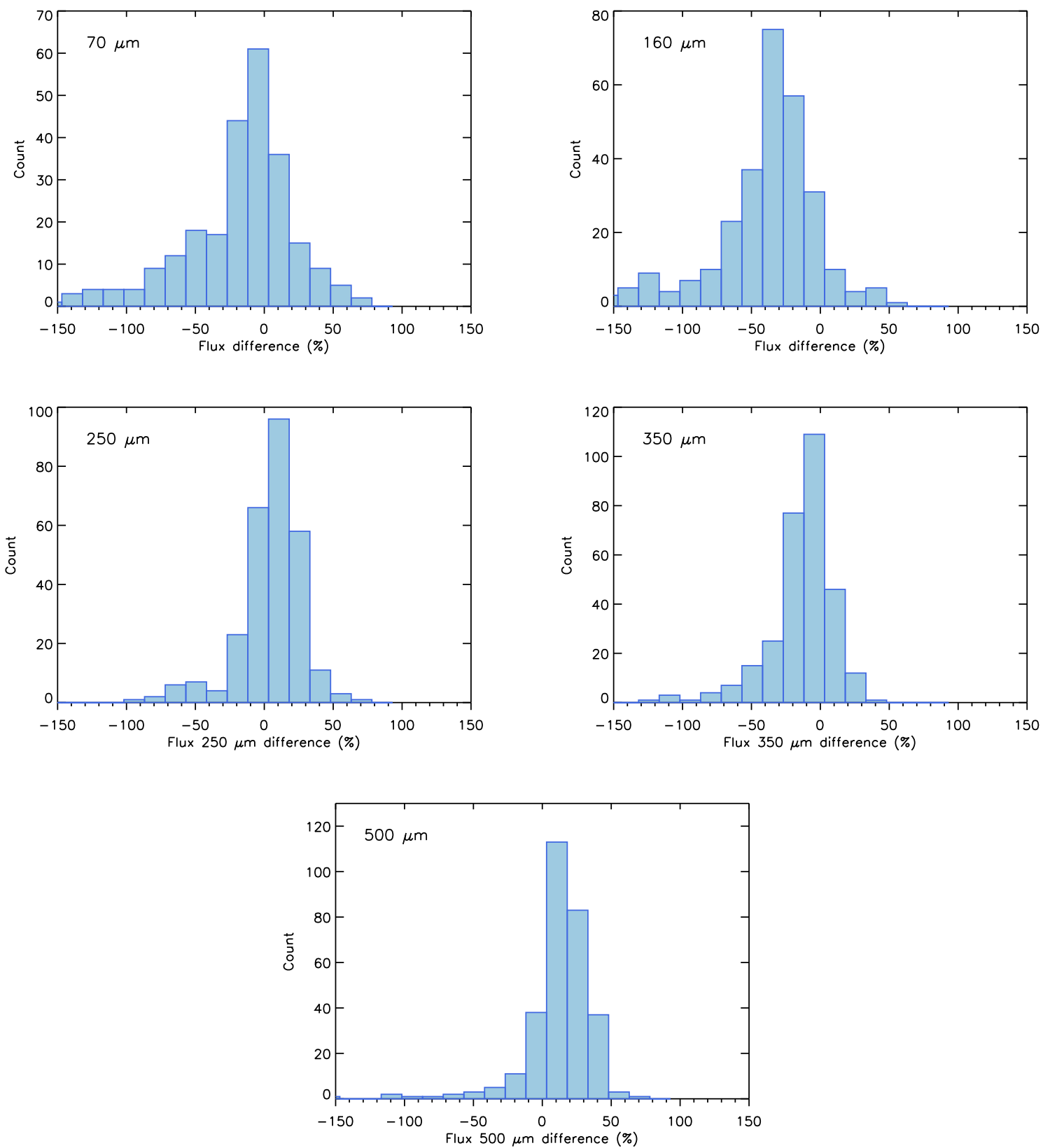

Figure A1. Difference in percentage between the fluxes of the clumps as estimated in Elia et al. (2017) and estimated using the Hyper algorithm. From the top: flux difference at 70, 160, 250, 350 and $500 \mu \mathrm{m}$, respectively.

highly variable backgrounds (Traficante et al. 2015b). The two FWHMs and the position angle of the clumps in the Elia et al. (2017) catalogue, estimated from the $250-\mu \mathrm{m}$ fit, have been used to define the radius of the ellipses over which we perform the Hyper aperture photometry at all wavelengths. This approach is substantially different from the method used in the Hi-GAL catalogue, as Cutex estimates the flux as the integral of the two-dimensional Gaussian fitted at each wavelength. The flux differences in percentage at each wavelength are shown in Fig. A1.

As expected, at the reference wavelength of $250 \mu \mathrm{m}$, the flux differences are minimal. At $\lambda<250 \mu \mathrm{m}$, the Cutex fluxes are lower than their Hyper counterparts, on average. This is particularly true at $160 \mu \mathrm{m}$, where the diffuse emission contributes substantially to the integrated flux. At $70 \mu \mathrm{m}$, the measured emission is dominated by the emission from the central protostars, and the differences are less sensitive to the photometry method. At $\lambda>250 \mu \mathrm{m}$, the Cutex fluxes are rescaled according to the $250-\mu \mathrm{m}$ size in the Hi-GAL catalogue (Elia et al. 2017), leading to small differences between the Hyper and Cutex photometry.

In order to add an additional point in the SED fitting, for each source we also extracted the fluxes at $870 \mu \mathrm{m}$, evaluated from the ATLASGAL calibrated maps using the same aperture adopted to extract the Hi-GAL fluxes.

Few sources (21) are saturated at $250 \mu \mathrm{m}$ in the Hi-GAL maps. This is because of a combination of the strong flux emission of some sources whose SED peaks at around the 160-250 $\mu \mathrm{m}$ wavelengths, and because of the higher dynamical range of the PACS instrument at $160 \mu \mathrm{m}$, which allows the $160-\mu \mathrm{m}$ band to saturate at higher 


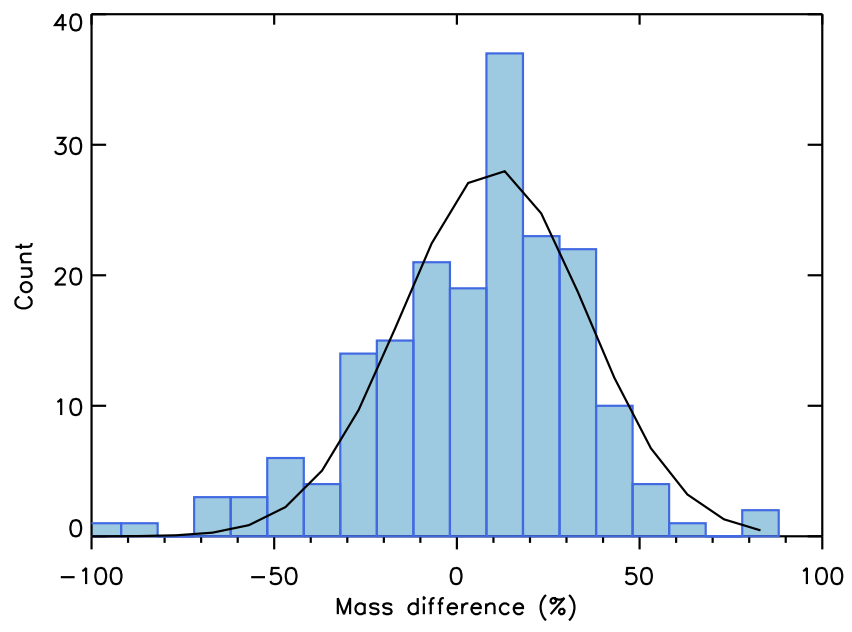

Figure A2. Mass differences (Cutex - Hyper values) for the 192 clumps with properties measured as in the Elia et al. (2017) catalogue and using Hyper. The black curve is the Gaussian fit to the distribution, used to estimate the uncertainties associated with the dust photometry.

fluxes than the SPIRE bands. While these sources still have a flux estimation in the Hi-GAL catalogue, no aperture photometry can be reliably performed, so we excluded these clumps from the comparison. The source properties have been evaluated using the same grey-body model described in Section 2 . We obtained a final sample of 192 clumps with well-defined dust properties that we used for the comparison. The mass differences in percentage are shown in Fig. A2.

To estimate the uncertainties on the mass due to the source photometry, we fit a Gaussian to the histogram of the mass differences. The standard deviation of the Gaussian is $\simeq 25$ per cent, which we assume as mass uncertainty. Note that the masses estimated with Hyper are $\simeq 10$ per cent systematically lower than their Cutex counterparts, likely as a consequence of the different photometry at $\lambda=160 \mu \mathrm{m}$. However, this systematic offset does not influence the main results of this work, as discussed in Section 2.4.

To further investigate this point, Fig. A 3 shows the $\alpha_{\text {vir }}-$ mass diagram and the mass-radius diagram obtained from the results of the Hyper photometry. The values of the slopes $\alpha$ and $\delta$ are
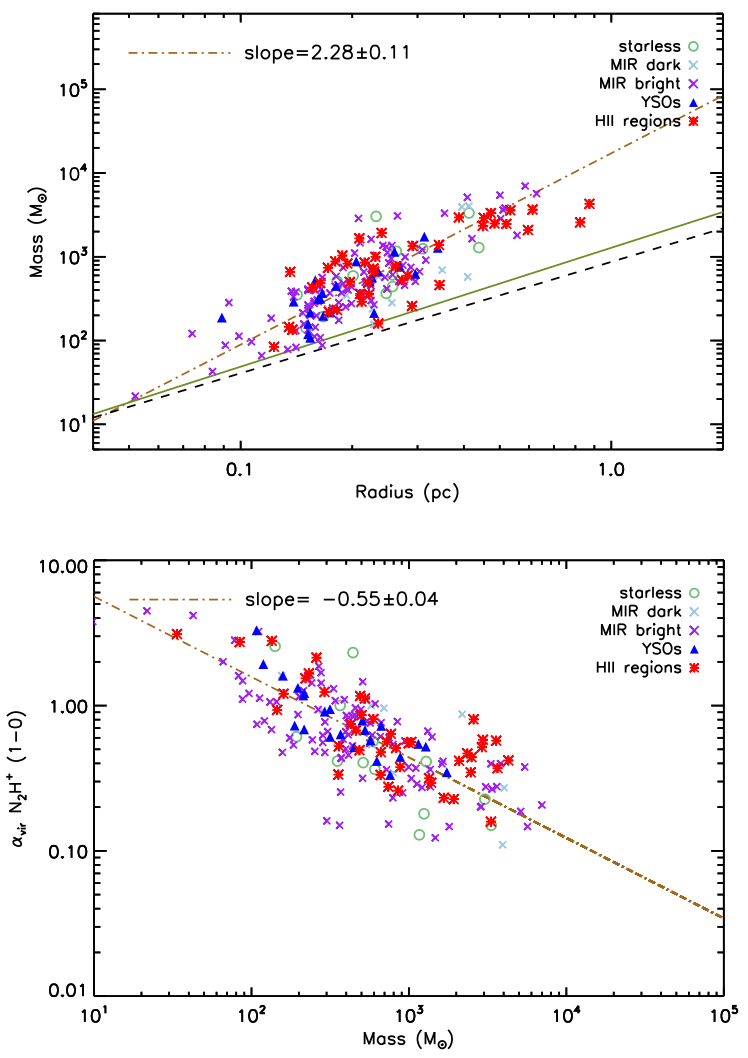

Figure A3. From the top: the same as in Figs 8 and 9 but assuming the dust parameters estimated using Hyper. The slopes of the diagrams are consistent with the findings discussed in the paper.

consistent with the values derived in Section 4.3. Also, the slope of the $\alpha_{\text {vir }}$-mass diagram is still determined from the slope of the mass-radius diagram, according to equation (2). Given a massradius slope of $\delta=2.28 \pm 0.11$, the expected $\alpha_{\text {vir }}$-mass slope would be $\alpha=-0.56 \pm 0.03$, in agreement with the result shown in Fig. A2 (i.e. $\alpha=-0.55 \pm 0.04$ ).

\section{APPENDIX B: CLUMP PROPERTIES}


Table B1. Properties of the 213 clumps analysed in this work. The clump radius is defined by the Cutex fit at $250 \mu \mathrm{m}$. The clump temperature, mass and luminosity are obtained from the SED fitting. $\Sigma$ is the clump surface density. $\sigma$ is the velocity dispersion obtained from the $\mathrm{N}_{2} \mathrm{H}^{+}(1-0)$ emission. The determination of the clump evolutionary phase is discussed in Section 3.

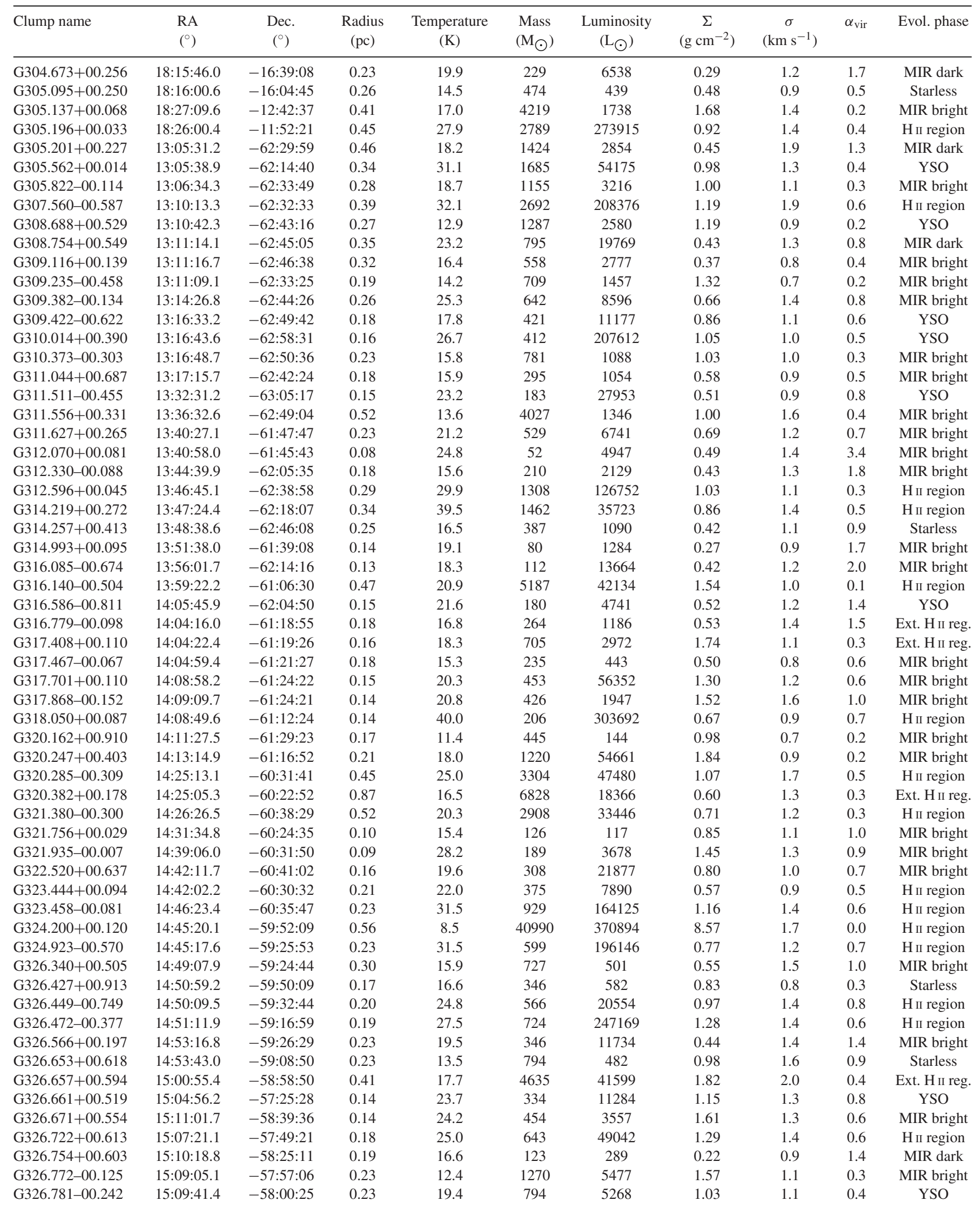


Table B1. continued

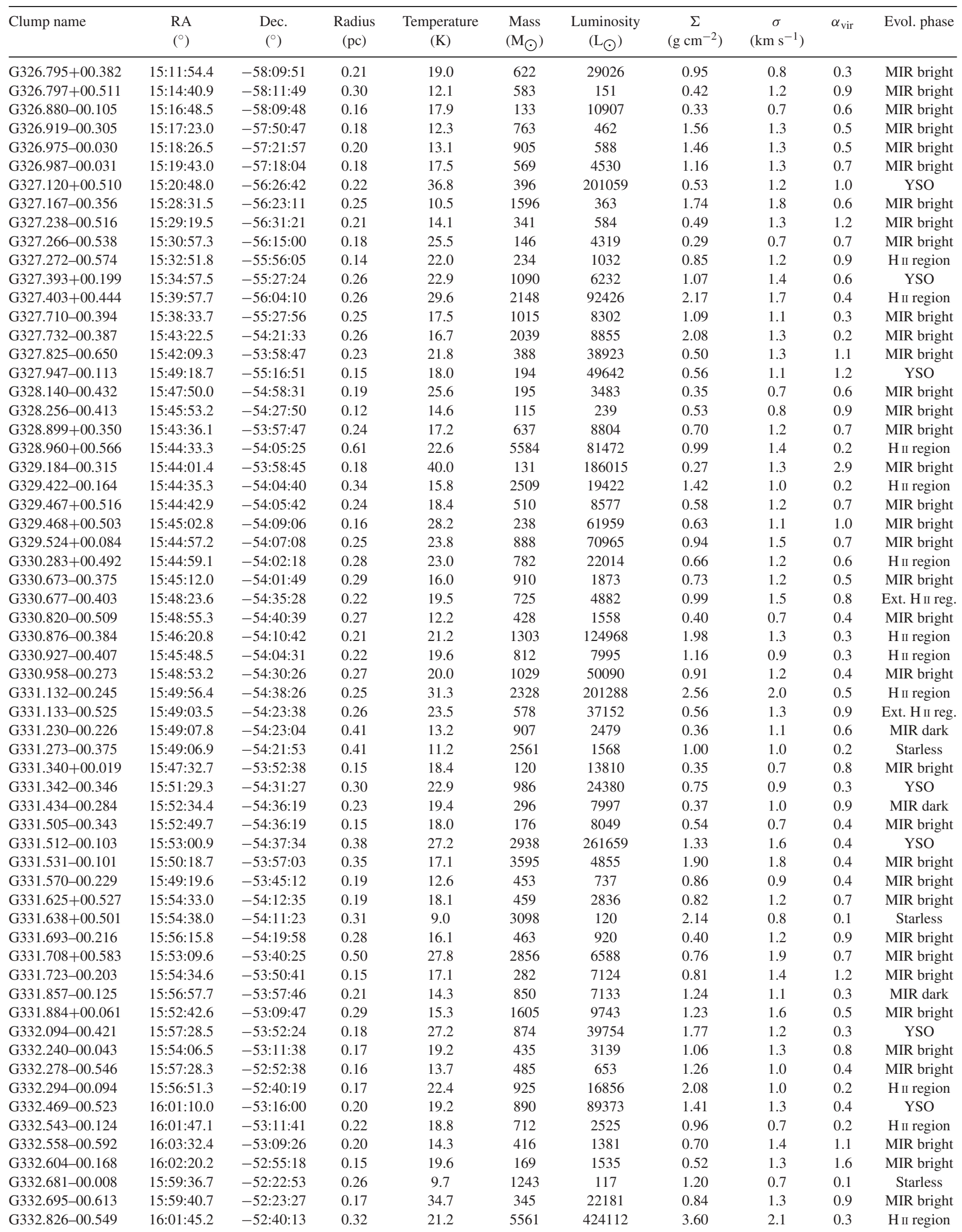


Table B1. continued

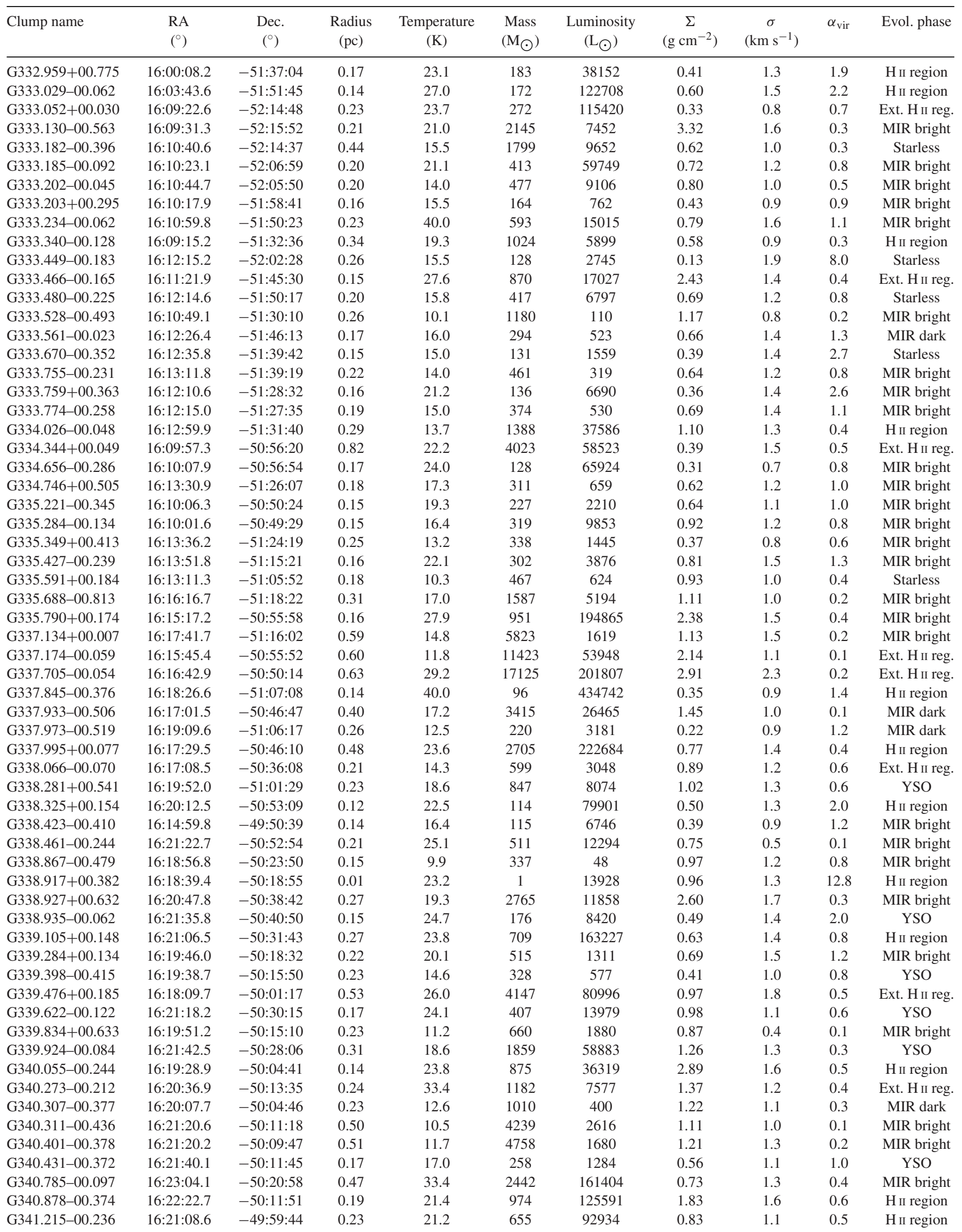


Table B1. continued

\begin{tabular}{|c|c|c|c|c|c|c|c|c|c|c|}
\hline Clump name & $\begin{array}{l}\text { RA } \\
\left({ }^{\circ}\right)\end{array}$ & $\begin{array}{c}\text { Dec. } \\
\left({ }^{\circ}\right)\end{array}$ & $\begin{array}{l}\text { Radius } \\
\text { (pc) }\end{array}$ & $\begin{array}{c}\text { Temperature } \\
\text { (K) }\end{array}$ & $\begin{array}{l}\text { Mass } \\
\left(\mathrm{M}_{\odot}\right)\end{array}$ & $\begin{array}{l}\text { Luminosity } \\
\qquad\left(\mathrm{L}_{\odot}\right)\end{array}$ & $\begin{array}{c}\Sigma \\
\left(\mathrm{g} \mathrm{cm}^{-2}\right)\end{array}$ & $\begin{array}{c}\sigma \\
\left(\mathrm{km} \mathrm{s}^{-1}\right)\end{array}$ & $\alpha_{\mathrm{vir}}$ & Evol. phase \\
\hline G341.218-00.213 & $16: 23: 02.8$ & $-50: 08: 55$ & 0.16 & 32.2 & 365 & 18395 & 0.96 & 1.4 & 1.0 & YSO \\
\hline G342.369+00.140 & $16: 20: 19.1$ & $-49: 34: 51$ & 0.05 & 19.4 & 27 & 22213 & 0.68 & 1.3 & 3.5 & MIR bright \\
\hline $\mathrm{G} 342.415+00.412$ & $16: 23: 06.0$ & $-50: 00: 36$ & 0.56 & 14.2 & 3398 & 10881 & 0.73 & 0.6 & 0.1 & MIR bright \\
\hline G342.484+00.183 & $16: 23: 17.2$ & $-49: 40: 59$ & 0.63 & 22.2 & 6698 & 19631 & 1.12 & 1.1 & 0.1 & MIR bright \\
\hline G342.706+00.125 & $16: 21: 37.1$ & $-49: 23: 28$ & 0.04 & 28.8 & 30 & 52852 & 1.60 & 1.6 & 3.4 & $\mathrm{H}$ II region \\
\hline G342.959-00.318 & $16: 27: 02.6$ & $-49: 24: 00$ & 0.17 & 20.6 & 213 & 44215 & 0.51 & 1.2 & 1.2 & YSO \\
\hline G343.134-00.484 & $16: 23: 58.3$ & $-48: 46: 58$ & 0.22 & 13.6 & 321 & 128 & 0.45 & 1.4 & 1.5 & MIR bright \\
\hline $\mathrm{G} 343.501+00.025$ & $16: 27: 26.2$ & $-49: 12: 34$ & 0.03 & 14.8 & 14 & 13364 & 1.41 & 1.1 & 2.6 & MIR bright \\
\hline G343.503-00.015 & $16: 29: 41.5$ & $-49: 01: 58$ & 0.18 & 23.2 & 719 & 461495 & 1.47 & 1.3 & 0.5 & $\mathrm{H}$ iा region \\
\hline G343.520-00.519 & $16: 29: 01.3$ & $-48: 50: 27$ & 0.16 & 21.3 & 348 & 3066 & 0.86 & 1.2 & 0.9 & YSO \\
\hline G343.689-00.018 & $16: 26: 55.0$ & $-48: 24: 58$ & 0.16 & 20.0 & 275 & 1224 & 0.71 & 1.0 & 0.6 & MIR bright \\
\hline G344.221-00.594 & $16: 35: 06.2$ & $-48: 46: 14$ & 0.16 & 34.1 & 336 & 37800 & 0.92 & 1.3 & 1.0 & $\mathrm{H}$ II region \\
\hline G344.246-00.670 & $16: 33: 29.5$ & $-48: 03: 43$ & 0.05 & 10.6 & 24 & 91 & 0.58 & 1.3 & 4.0 & Starless \\
\hline G344.726-00.541 & $16: 34: 13.2$ & $-48: 06: 15$ & 0.14 & 10.2 & 292 & 50 & 0.95 & 0.9 & 0.5 & Starless \\
\hline G345.132-00.175 & $16: 34: 11.1$ & $-47: 33: 24$ & 0.09 & 18.4 & 98 & 747 & 0.80 & 1.1 & 1.3 & MIR bright \\
\hline G345.144-00.217 & $16: 34: 38.7$ & $-47: 36: 28$ & 0.11 & 16.8 & 107 & 896 & 0.62 & 1.0 & 1.1 & MIR bright \\
\hline $\mathrm{G} 345.718+00.818$ & $16: 33: 40.1$ & $-47: 23: 28$ & 0.09 & 18.5 & 207 & 1069 & 1.75 & 1.1 & 0.7 & YSO \\
\hline G346.078-00.056 & $16: 36: 17.2$ & $-47: 40: 46$ & 0.60 & 21.8 & 4806 & 83689 & 0.89 & 1.5 & 0.3 & $\mathrm{H}$ in region \\
\hline G347.967-00.434 & $16: 35: 58.7$ & $-47: 23: 36$ & 0.41 & 18.9 & 4779 & 118251 & 1.86 & 1.5 & 0.2 & MIR dark \\
\hline G348.171+00.465 & $16: 36: 18.9$ & $-47: 23: 17$ & 0.07 & 16.7 & 168 & 390 & 2.03 & 1.1 & 0.6 & MIR bright \\
\hline $\mathrm{G} 348.181+00.482$ & $16: 36: 25.5$ & $-47: 24: 26$ & 0.08 & 22.1 & 337 & 1448 & 3.41 & 1.0 & 0.3 & MIR bright \\
\hline G349.092+00.106 & $16: 36: 15.4$ & $-47: 19: 02$ & 0.47 & 40.0 & 1277 & 142104 & 0.38 & 1.7 & 1.2 & MIR bright \\
\hline
\end{tabular}



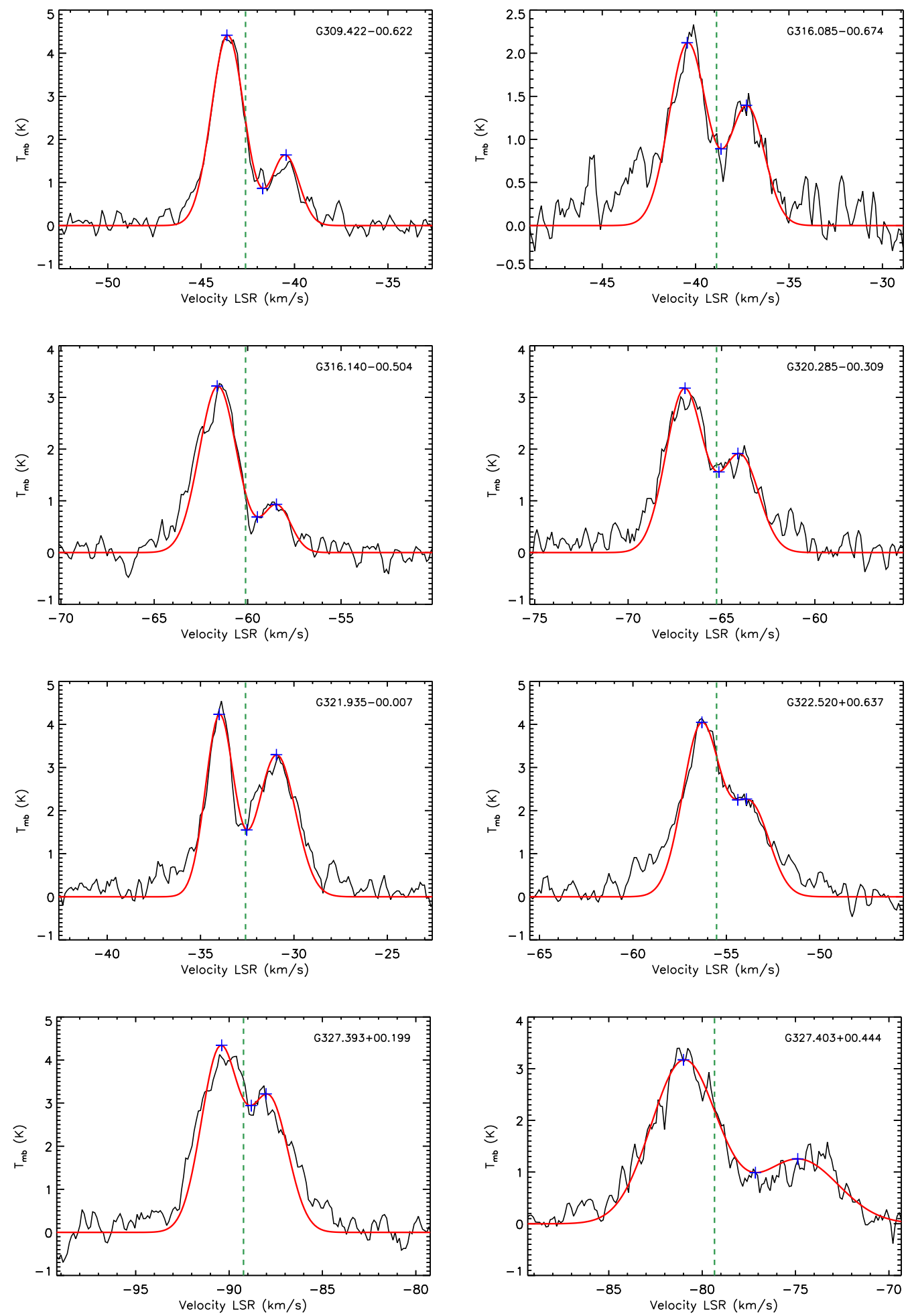

Figure C1. $\mathrm{HCO}^{+}(1-0)$ spectra of the 21 clumps with double-peaked blue asymmetries. The red lines in Fig. $\mathrm{C} 1$ are the double-Gaussian fits, and the blue crosses are the positions of the two identified peaks corresponding to the dip between the peaks. The green-dashed lines are in correspondence of the central velocity of each clump, determined from the $\mathrm{N}_{2} \mathrm{H}^{+}(1-0)$ spectra. 

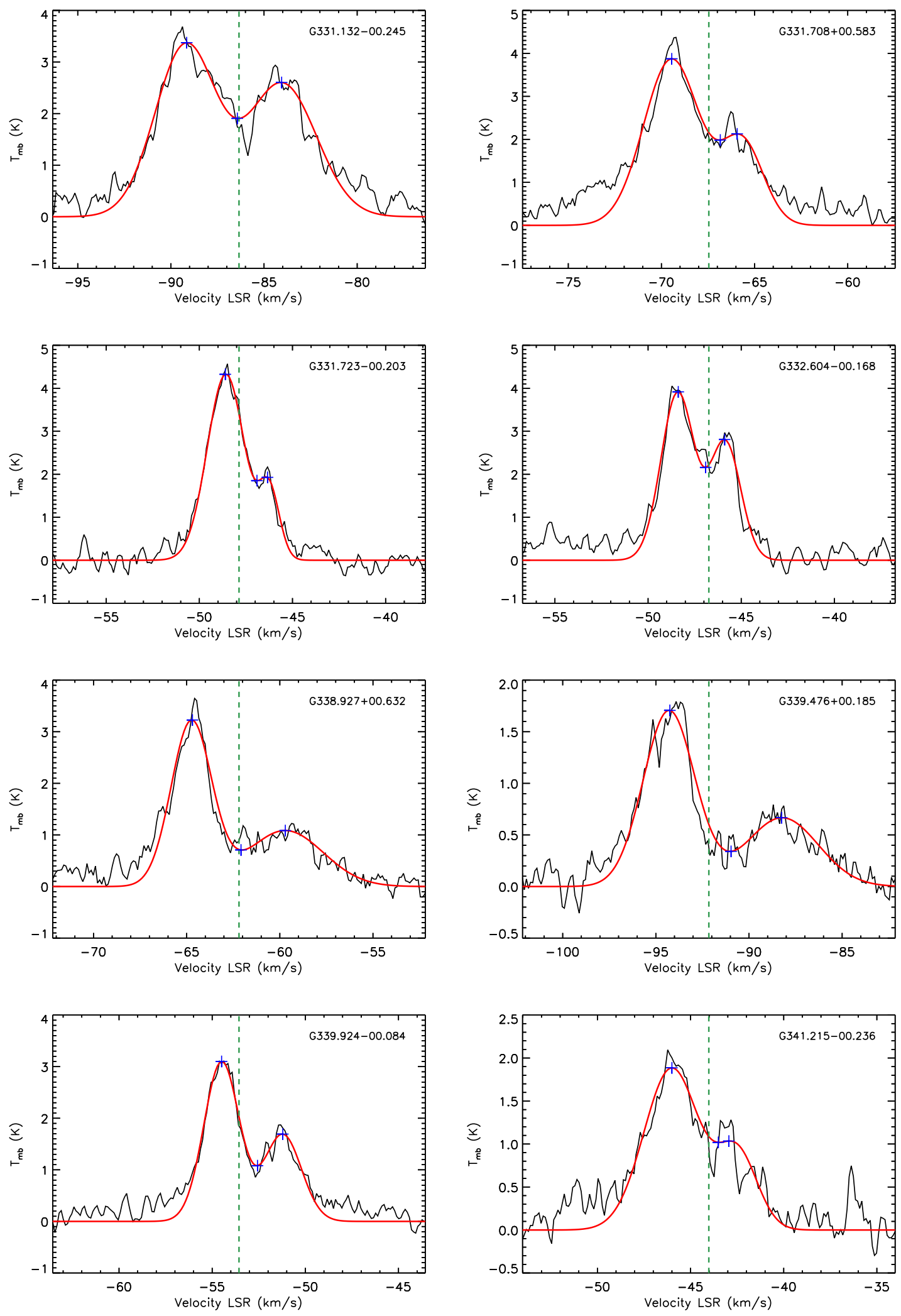

Figure C1. continued 

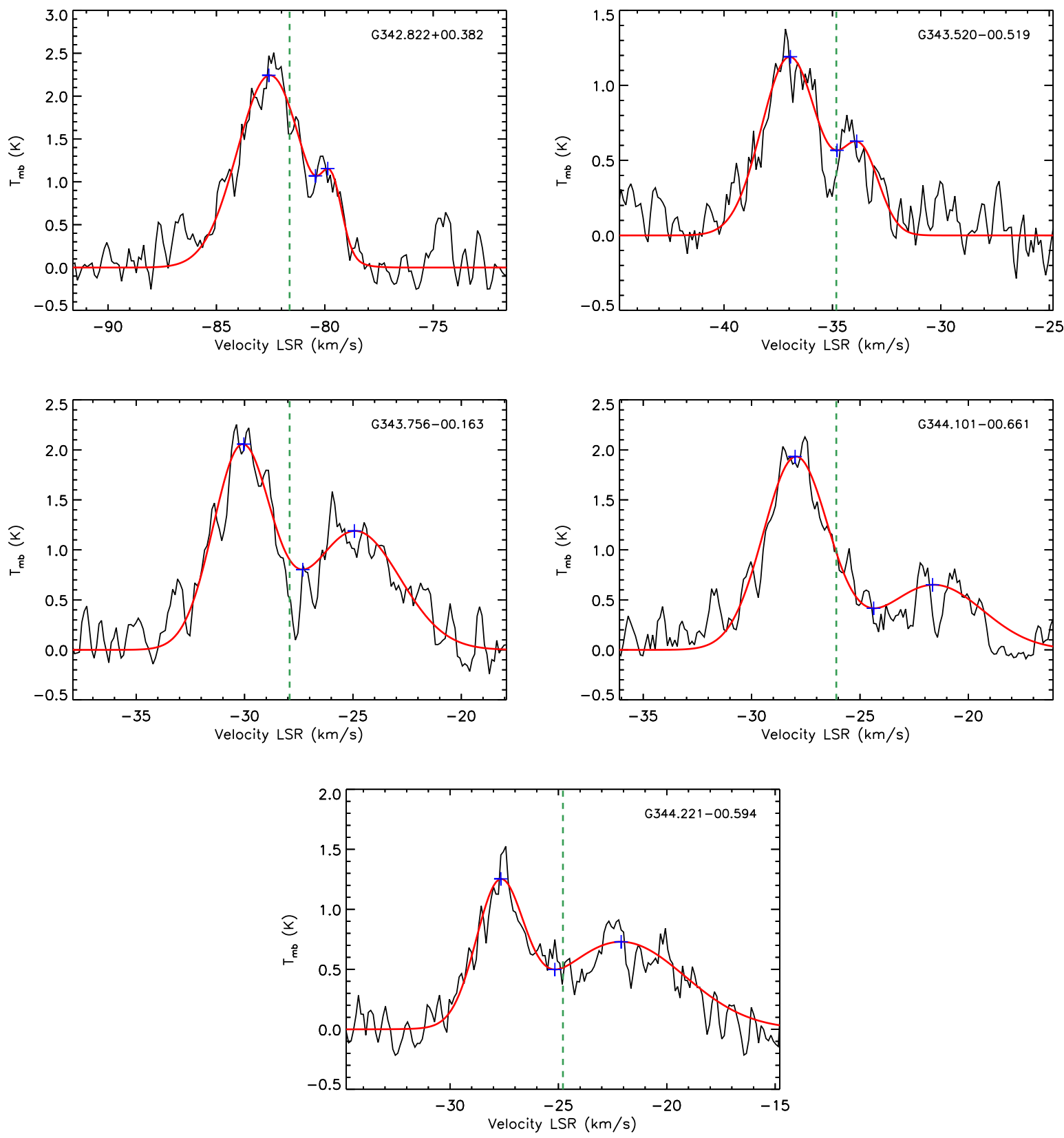

Figure C1. continued

This paper has been typeset from a $\mathrm{T}_{\mathrm{E}} \mathrm{X} / \mathrm{LAT}_{\mathrm{E}} \mathrm{X}$ file prepared by the author. 